\title{
TITLE:
}

\section{Electronic energy density in chemical reaction systems}

$\operatorname{AUTHOR}(\mathrm{S})$ :

Tachibana, A

\section{CITATION:}

Tachibana, A. Electronic energy density in chemical reaction systems. JOURNAL OF CHEMICAL PHYSICS 2001, 115(8): 3497-3518

\section{ISSUE DATE:}

2001-08-22

URL:

http://hdl.handle.net/2433/43521

\section{RIGHT:}

Copyright 2001 American Institute of Physics. This article may be downloaded for personal use only. Any other use requires prior permission of the author and the American Institute of Physics. 


\title{
Electronic energy density in chemical reaction systems
}

\author{
Akitomo Tachibana ${ }^{\text {a) }}$ \\ Department of Engineering Physics and Mechanics, Kyoto University, Kyoto 606-8501, Japan
}

(Received 4 December 2000; accepted 17 May 2001)

The energy of chemical reaction is visualized in real space using the electronic energy density $n_{E}(\vec{r})$ associated with the electron density $n(\vec{r})$. The electronic energy density $n_{E}(\vec{r})$ is decomposed into the kinetic energy density $n_{T}(\vec{r})$, the external potential energy density $n_{V}(\vec{r})$, and the interelectron potential energy density $n_{W}(\vec{r})$. Using the electronic energy density $n_{E}(\vec{r})$ we can pick up any point in a chemical reaction system and find how the electronic energy $E$ is assigned to the selected point. We can then integrate the electronic energy density $n_{E}(\vec{r})$ in any region $R$ surrounding the point and find out the regional electronic energy $E_{R}$ to the global $E$. The kinetic energy density $n_{T}(\vec{r})$ is used to identify the intrinsic shape of the reactants, the electronic transition state, and the reaction products along the course of the chemical reaction coordinate. The intrinsic shape is identified with the electronic interface $S$ that discriminates the region $R_{D}$ of the electronic drop from the region $R_{A}$ of the electronic atmosphere in the density distribution of the electron gas. If the $R$ spans the whole space, then the integral gives the total $E$. The regional electronic energy $E_{R}$ in thermodynamic ensemble is realized in electrochemistry as the intrinsic Volta electric potential $\phi_{R}$ and the intrinsic Herring-Nichols work function $\Phi_{R}$. We have picked up first a hydrogen-like atom for which we have analytical exact expressions of the relativistic kinetic energy density $n_{T_{M}}(\vec{r})$ and its nonrelativistic version $n_{T}(\vec{r})$. These expressions are valid for any excited bound states as well as the ground state. Second, we have selected the following five reaction systems and show the figures of the $n_{T}(\vec{r})$ as well as the other energy densities along the intrinsic reaction coordinates: a protonation reaction to $\mathrm{He}$, addition reactions of $\mathrm{HF}$ to $\mathrm{C}_{2} \mathrm{H}_{4}$ and $\mathrm{C}_{2} \mathrm{H}_{2}$, hydrogen abstraction reactions of $\mathrm{NH}_{3}^{+}$ from $\mathrm{HF}$ and $\mathrm{NH}_{3}$. Valence electrons possess their unique delocalized drop region remote from those heavily localized drop regions adhered to core electrons. The kinetic energy density $n_{T}(\vec{r})$ and the tension density $\vec{\tau}^{S}(\vec{r})$ can vividly demonstrate the formation of the chemical bond. Various basic chemical concepts in these chemical reaction systems have been clearly visualized in real three-dimensional space. (C) 2001 American Institute of Physics. [DOI: 10.1063/1.1384012]

\section{INTRODUCTION}

The redistribution of electrons is essential in the course of chemical reaction coordinates. ${ }^{1-3}$ This is because the associated lowering in the electronic energy is the driving force of chemical reaction. The decomposition of the electronic energy in the abstract functional space of orbital has played a significant role in the study of chemical reactivity indices such as Coulson valence bond theory, Woodward-Hoffmann law, and the Fukui frontier orbital theory. ${ }^{4-6}$

We have recently developed a novel theory of energy decomposition in the real space. ${ }^{7-10}$ The new energy decomposition scheme is exact and complementary to the conventional orbital-space energy decomposition scheme. Namely, which region of space has significant contribution to chemical reaction coordinate is easily recognized. This is advantageous in visualization of the chemical interaction in real space.

This new regional energy decomposition scheme is here in the present paper extended to an infinitely small regionalenergy decomposition scheme, namely, the electronic energy density decomposition scheme. Our scheme here presented enables us to recognize how the electronic energy density is

${ }^{a)}$ Electronic mail: akitomo@scl.kyoto-u.ac.jp associated with the electron density. Using the electronic energy density, we can pick up any point in a chemical reaction system and find how the electronic energy $E$ is assigned to the point. We can then integrate the electronic energy density in a small region and find out the regional electronic energy contribution to the global electronic energy $E$. If the integration spans the whole space, then the integral gives the total $E$. We assume in this paper the adiabatic Born-Oppenheimer treatment for the electronic structure. All the calculations are performed at the HF level of theory. The basis set is 6-311G** unless otherwise stated explicitly.

\section{REGIONAL ENERGY DECOMPOSITION}

\section{A. Electronic energy density}

According to Heisenberg's uncertainty principle, we cannot know the position of an electron precisely but we merely know the electron density $n(\vec{r})$ if the energy of the quantum mechanical state of the electron gas is given. The electron density $n(\vec{r})$ plays an important role in newly developed density functional theory of materials. ${ }^{11-13}$ In chemical reaction systems, the redistribution of electrons directly redefines the electronic energy $E$ as a unique functional of the electron density $n(\vec{r})$. 
In Ref. 8, we have developed a novel field theory of regional energy decomposition. Here we shall briefly review the theory ${ }^{8}$ augmented by the new definition of the kinetic energy density operator rigged with gauge invariance of the theory. The gauge invariance is essential to the theory of "density" as well as the integrated quantity such as energy. This is because the gauge merely changes the phase of the wave function:

$$
\psi(\vec{r}) \rightarrow \psi^{\prime}(\vec{r})=\exp \left(-i \frac{e}{\hbar c} \theta(\vec{r})\right) \psi(\vec{r}) .
$$

The phase itself should not affect the density as well as the integrated quantity such as total energy. Since the gradient of the wave function should appear in the equation of motion of electrons, the gauge invariance of theory should inevitably require the gauge potential. The gradient operator should then change to covariant derivative operator. The gauge potential brings about the gauge field, namely the electromagnetic field. Thus the introduction of the electromagnetic field is mandatory by the gauge invariance. This kind of gauge principle has been fundamental in the modern quantum theory of electrons.

For the simplicity of presentation of theory, full relativistic gauge invariant theory of quantum electrodynamics (QED) will be presented in Sec. II B [see also Appendices A and B; specifically Eqs. (B12) and (B13)].

In Sec. II A, we shall first start from the nonrelativistic electronic field theory. For the nonrelativistic electronic field theory the Lagrangian is invariant under the gauge transformation, and the Hamiltonian density operator $\hat{H}(\vec{r})$ is given using the covariant derivative as follows:

$$
\hat{H}(\vec{r})=\hat{T}(\vec{r})+\hat{V}(\vec{r})+\hat{W}(\vec{r}),
$$

where $\hat{T}(\vec{r})$ is the kinetic energy density operator, $\hat{V}(\vec{r})$ the external potential energy density operator associated with the external electrostatic potential $v(\vec{r})=-e \phi(\vec{r})$ (see Appendix A), while $\hat{W}(\vec{r})$ denotes interelectron potential energy density operator associated with the interelectron potential energy,

$$
\begin{aligned}
w(\vec{r}, \vec{s})= & \frac{e^{2}}{|\vec{r}-\vec{s}|}: \\
\hat{T}(\vec{r})= & -\frac{\hbar^{2}}{2 m} \cdot \frac{1}{2}\left(\hat{\chi}^{+}(\vec{r}) D_{k}^{2}(\vec{r}) \hat{\chi}(\vec{r})\right. \\
& \left.+D_{k}^{* 2}(\vec{r}) \hat{\chi}^{+}(\vec{r}) \cdot \hat{\chi}(\vec{r})\right), \\
\hat{V}(\vec{r})=\hat{\chi}^{+}(\vec{r}) \nu(\vec{r}) \hat{\chi}(\vec{r})=-e \hat{\chi}^{+}(\vec{r}) \phi(\vec{r}) \hat{\chi}(\vec{r}), & \\
\hat{W}(\vec{r})= & \frac{1}{2} \hat{\chi}^{+}(\vec{r}) \int \mathrm{d}^{3} \vec{s} \hat{\chi}^{+}(\vec{s}) w(\vec{r}, \vec{s}) \hat{\chi}(\vec{s}) \cdot \hat{\chi}(\vec{r}),
\end{aligned}
$$

where $D_{k}(\vec{r})$ denotes the covariant derivative (see Appendix A) and $\hat{\chi}(\vec{r})$ denotes the three-dimensional two-component spinor operator,

$$
\hat{\chi}(\vec{r})=\left(\begin{array}{l}
\hat{\chi}^{1}(\vec{r}) \\
\hat{\chi}^{2}(\vec{r})
\end{array}\right)
$$

(see Appendix B).
For electron density operator

$$
\hat{N}(\vec{r})=\hat{\chi}^{+}(\vec{r}) \hat{\chi}(\vec{r}),
$$

the probability conservation is represented as the continuity equation,

$$
\frac{\partial}{\partial t} \hat{N}(\vec{r})+\operatorname{div} \hat{\vec{S}}(\vec{r})=0,
$$

where the velocity density operator,

$$
\begin{aligned}
\hat{S}^{k}(\vec{r})= & \frac{1}{2 m}\left(-i \hbar \hat{\chi}^{+}(\vec{r}) D_{k}(\vec{r}) \hat{\chi}(\vec{r})\right. \\
& \left.+i \hbar D_{k}^{*}(\vec{r}) \hat{\chi}^{+}(\vec{r}) \cdot \hat{\chi}(\vec{r})\right),
\end{aligned}
$$

satisfies the equation of motion,

$$
m \frac{\partial}{\partial t} \hat{\vec{S}}(\vec{r})=\hat{\vec{\tau}} S(\vec{r})+\hat{\vec{K}}(\vec{r})-\frac{e}{c} \hat{\vec{S}}(\vec{r}) \times \vec{B}(\vec{r}) .
$$

Aside from the Lorentz force density operator, the stress vector density operator or the tension density operator $\hat{\vec{\tau}}^{S}(\vec{r})$ is given as the divergence of the stress tensor density operator $\hat{\vec{\tau}} S(\vec{r})$ as follows:

$$
\hat{\tau}^{S k}(\vec{r})=\partial_{l} \hat{\tau}^{S k l}(\vec{r}),
$$

where

$$
\begin{aligned}
\hat{\tau}^{S k}(\vec{r})= & \frac{\hbar^{2}}{4 m}\left[\hat{\chi}^{+}(\vec{r}) D_{k}(\vec{r}) D_{l}^{2}(\vec{r}) \hat{\chi}(\vec{r})\right. \\
& +D_{k}^{*}(\vec{r}) D_{l}^{* 2}(\vec{r}) \hat{\chi}^{+}(\vec{r}) \cdot \hat{\chi}(\vec{r}) \\
& -D_{k}^{*}(\vec{r}) \hat{\chi}^{+}(\vec{r}) \cdot D_{l}^{2}(\vec{r}) \hat{\chi}(\vec{r}) \\
& \left.-D_{l}^{* 2}(\vec{r}) \hat{\chi}^{+}(\vec{r}) \cdot D_{k}(\vec{r}) \hat{\chi}(\vec{r})\right] \\
& +\frac{e}{c}(\vec{S}(\vec{r}) \times \vec{B}(\vec{r}))^{k},
\end{aligned}
$$

and

$$
\begin{aligned}
\hat{\tau}^{S k l}(\vec{r})= & \frac{\hbar^{2}}{4 m}\left[\hat{\chi}^{+}(\vec{r}) D_{k}(\vec{r}) D_{l}(\vec{r}) \hat{\chi}(\vec{r})\right. \\
& +D_{k}^{*}(\vec{r}) D_{l}^{*}(\vec{r}) \hat{\chi}^{+}(\vec{r}) \cdot \hat{\chi}(\vec{r}) \\
& -D_{k}^{*}(\vec{r}) \hat{\chi}^{+}(\vec{r}) \cdot D_{l}(\vec{r}) \hat{\chi}(\vec{r}) \\
& \left.-D_{l}^{*}(\vec{r}) \hat{\chi}^{+}(\vec{r}) \cdot D_{k}(\vec{r}) \hat{\chi}(\vec{r})\right] .
\end{aligned}
$$

Second, the force density operator $\widehat{\vec{K}}(\vec{r})$ is given as

$$
\begin{aligned}
\hat{K}^{k}(\vec{r})= & -\hat{\chi}^{+}(\vec{r})\left(\partial_{k} \nu(\vec{r})+\int d^{3} \vec{s} \hat{\chi}^{+}(\vec{s}) \partial_{k} w(\vec{r}, \vec{s}) \hat{\chi}(\vec{s})\right) \\
& \cdot \hat{\chi}(\vec{r})+\frac{e}{c} \frac{\partial A^{k}(\vec{r})}{\partial t} \hat{N}(\vec{r}) .
\end{aligned}
$$

Let the expectation value over the ensemble associated with the density matrix $\Sigma_{\alpha}\left|\Psi_{\alpha}\right\rangle p_{\alpha}\left\langle\Psi_{\alpha}\right|$ in the Fock space be denoted as \langle\rangle such as 


$$
\begin{aligned}
& \langle\hat{N}(\vec{r})\rangle=\sum_{\alpha} p_{\alpha}\left\langle\Psi_{\alpha}|\hat{N}(\vec{r})| \Psi_{\alpha}\right\rangle, \quad\left\langle\Psi_{\alpha} \mid \Psi_{\alpha}\right\rangle=1, \\
& \sum_{\alpha} p_{\alpha}=1, \quad 1 \geqslant p_{\alpha}>0,
\end{aligned}
$$

then the electron density $n(\vec{r})$ is given as

$$
n(\vec{r})=\langle\hat{N}(\vec{r})\rangle .
$$

It should be noted that the choice of the vacuum and state vectors and the ensemble itself should reflect the proper physics of the system we treat. Then, we obtain the energy densities as follows:

$$
\begin{aligned}
& n_{E}(\vec{r})=\langle\hat{H}(\vec{r})\rangle, \\
& n_{T}(\vec{r})=\langle\hat{T}(\vec{r})\rangle, \\
& n_{V}(\vec{r})=\langle\hat{V}(\vec{r})\rangle, \\
& n_{W}(\vec{r})=\langle\hat{W}(\vec{r})\rangle .
\end{aligned}
$$

The kinetic energy density $n_{T}(\vec{r})$, the external potential energy density $n_{V}(\vec{r})$, and the interelectron potential energy density $n_{W}(\vec{r})$ are not mutually independent but are related to each other because they are all derived from the same density matrix. Thus, the three components should follow the sum rule leading to the total energy density $n_{E}(\vec{r})$ :

$$
n_{E}(\vec{r})=n_{T}(\vec{r})+n_{V}(\vec{r})+n_{W}(\vec{r}) .
$$

Regional energy decomposition ${ }^{7-9}$ is found to be the integration over region $R$ of space as follows:

$$
\begin{aligned}
& E_{R}=\int_{\vec{r} \in R} d^{3} \vec{r} n_{E}(\vec{r}), \\
& T_{R}=\int_{\vec{r} \in R} d^{3} \vec{r} n_{T}(\vec{r}), \\
& V_{R}=\int_{\vec{r} \in R} d^{3} \vec{r} n_{V}(\vec{r}), \\
& W_{R}=\int_{\vec{r} \in R} d^{3} \vec{r} n_{W}(\vec{r}),
\end{aligned}
$$

where $\vec{r} \in R$ means the regional integral confined within the region $R$ exclusively. Apparently, we have the sum rule of the regional energies as follows:

$$
E_{R}=T_{R}+V_{R}+W_{R} .
$$

If the whole space is decomposed into a set of regions, then we obtain ${ }^{7}$

$$
E=\sum_{R} E_{R}
$$

where $\Sigma_{R}$ denotes summation over the set of regions and where the electron energy $E$ is the expectation value of the electron Hamiltonian operator in the whole space:

$$
E=\langle\hat{H}\rangle, \quad \hat{H}=\int d^{3} \vec{r} \hat{H}(\vec{r}) .
$$

Likewise, the electron number $N$ is defined and decomposed regionally as follows:

$$
\begin{aligned}
& N=\langle\hat{N}\rangle, \quad \hat{N}=\int d^{3} \vec{r} \hat{N}(\vec{r}), \\
& N=\sum_{R} N_{R}, \quad N_{R}=\int_{\vec{r} \in R} d^{3} \vec{r} n(\vec{r}) .
\end{aligned}
$$

It should be noted that the choice of the new kinetic energy density operator $\hat{T}(\vec{r})$ is not positive definite. Conventional positive-semidefinite choice $\hat{T}_{\text {conv }}(\vec{r})$ reads

$$
\hat{T}_{\text {conv }}(\vec{r})=\frac{\hbar^{2}}{2 m} D_{k}^{*}(\vec{r}) \hat{\chi}^{+}(\vec{r}) \cdot D_{k}(\vec{r}) \hat{\chi}(\vec{r}) .
$$

Both expectation values in the whole space are the same: ${ }^{8}$

$$
\int d^{3} \vec{r}\left\langle\hat{T}_{\text {conv }}(\vec{r})\right\rangle=\int d^{3} \vec{r}\langle\hat{T}(\vec{r})\rangle,
$$

but the density is different from each other: $\hat{T}_{\text {conv }}(\vec{r})$ $\neq \hat{T}(\vec{r})$. In our previous calculations, ${ }^{8-10}$ which choice of the kinetic energy densities is appropriate has not been explicitly emphasized. But this difference is very important when we study the behavior of the newly defined kinetic energy density $n_{T}(\vec{r})$ in chemical reaction systems as follows.

In the very vicinity of atomic nucleus, the electron feels infinitely large positive electric potential of the bare nucleus. Then, in terms of classical mechanics, the electron that has constant energy can acquire infinitely large positive kinetic energy at the position of the nucleus. In terms of quantum mechanics as well, the $n_{T}(\vec{r})$ should then become infinitely positive at the position of nucleus, provided that, which is the very case of normal chemistry without radiation field, the intramolecular electric field $\vec{E}_{\text {intra }}(\vec{r})$ produced by the other electrons does not exceed that of the bare nucleus. ${ }^{14-16}$ The nucleus is therefore normally surrounded by the surface of zero kinetic energy density, $n_{T}(\vec{r})=0$, within which the kinetic energy density $n_{T}(\vec{r})>0$ where the electron density is amply accumulated and classically allowed motion of electron is guaranteed. We may call this the region of the electronic drop denoted by $R_{D}$ and the complementary region the region of the electronic atmosphere denoted by $R_{A}$, being separated by the electronic interface $S$ :

$$
\begin{aligned}
& R_{D}=\left\{\vec{r} \mid n_{T}(\vec{r})>0\right\}, \quad R_{A}=\left\{\vec{r} \mid n_{T}(\vec{r})<0\right\}, \\
& S=\left\{\vec{r} \mid n_{T}(\vec{r})=0\right\} .
\end{aligned}
$$

In the $R_{A}$ the electron density is dried up and the motion of electron is classically forbidden and the boundary $S$ in between $R_{D}$ and $R_{A}$ gives a clear image of the intrinsic shape of the reactant atoms and molecules, the reaction intermediates, and the reaction products along the course of the chemical reaction coordinate. In Sec. III, we shall demonstrate the usefulness of this new concept.

\section{B. Coulson conjecture of energy density}

In his book, Coulson conjectured that the physical meaning of the probability density should be related with the energy density of the wave equation in general. Coulson is the first chemist to envision the intimacy of the field theoretical 
energy density both of photons and electrons. As a matter of fact, for radiation field with electric field $\vec{E}(\vec{r})$ and magnetic field $\vec{B}(\vec{r})$ the radiation field energy density is $(1 / 8 \pi)$ $\times\left(\vec{E}^{2}(\vec{r})+\vec{B}^{2}(\vec{r})\right) .{ }^{4}$ We have realized the Coulson conjecture in such a way that without the radiation field the quantum electronic Hamiltonian density $n_{E}(\vec{r})$ for the ground state is manifestly given as the exact functional of electron density $n(\vec{r})^{7,11}$ as presented in Eq. (20) in Sec. II A. The inclusion of the radiation field to the electron field is to prove the self-consistent view to the Coulson conjecture. This is established in terms of the gauge principle of quantum electrodynamics (QED). The relativistic QED Lagrangian composed of the quantized electromagnetic and electronic fields is invariant under the Lorentz and gauge transformation.

The relativistic Dirac Hamiltonian density operator of electrons $\hat{H}_{\text {Dirac }}(\vec{r})$ is composed of the mass density operator $\hat{M}(\vec{r})$ and the external potential energy density operator $-e \hat{\Phi}(\vec{r})$ (see Appendices A and B):

$$
\begin{aligned}
& \hat{H}_{\text {Dirac }}(\vec{r})=\hat{M}(\vec{r})-e \hat{\Phi}(\vec{r}), \\
& \hat{M}(\vec{r})=\hat{\psi}^{+}(\vec{r})\left(-i \hbar c \gamma^{0} \gamma^{k} \hat{D}_{k}(\vec{r})+\gamma^{0} m c^{2}\right) \hat{\psi}(\vec{r}), \\
& \hat{\Phi}(\vec{r})=\hat{\psi}^{+}(\vec{r}) \hat{\phi}(\vec{r}) \hat{\psi}(\vec{r}) .
\end{aligned}
$$

In this expression, $\hat{\psi}(\vec{r})$ denotes the four-dimensional fourcomponent spinor operator,

$$
\hat{\psi}(\vec{r})=\left(\begin{array}{c}
\hat{\psi}_{1}(\vec{r}) \\
\hat{\psi}_{2}(\vec{r}) \\
\hat{\psi}_{3}(\vec{r}) \\
\hat{\psi}_{4}(\vec{r})
\end{array}\right) .
$$

The charge conservation is represented as the continuity equation,

$$
\partial_{\mu} \hat{j}^{\mu}(\vec{r})=\frac{\partial \hat{\rho}(\vec{r})}{\partial t}+\operatorname{div} \hat{\vec{j}}(\vec{r})=0,
$$

where the electronic charge density operator $\hat{\rho}(\vec{r})$,

$$
\hat{\rho}(\vec{r})=-e \hat{\psi}^{+}(\vec{r}) \hat{\psi}(\vec{r})=-e \hat{\bar{\psi}}(\vec{r}) \gamma^{0} \hat{\psi}(\vec{r})=\frac{1}{c} \hat{j}^{0}(\vec{r}),
$$

and the electronic current density operator $\hat{\vec{j}}(\vec{r})$,

$$
\hat{\vec{j}}(\vec{r})=-c e \hat{\psi}^{+}(\vec{r}) \gamma^{0} \vec{\gamma} \hat{\psi}(\vec{r})=-c e \hat{\bar{\psi}}(\vec{r}) \vec{\gamma} \hat{\psi}(\vec{r}),
$$

construct four-vector of momentum density operator,

$$
\hat{j}^{\mu}(\vec{r})=\left(\hat{j}^{0}(\vec{r}), \hat{\vec{j}}(\vec{r})\right)
$$

The kinetic momentum density operator $\widehat{\widehat{\Pi}}(\vec{r})$,

$$
\begin{aligned}
\hat{\Pi}^{k}(\vec{r})= & \frac{1}{2}\left(-i \hbar \hat{\psi}^{+}(\vec{r}) \hat{D}_{k}(\vec{r}) \hat{\psi}(\vec{r})\right. \\
& \left.+i \hbar \hat{D}_{k}^{+}(\vec{r}) \hat{\psi}^{+}(\vec{r}) \cdot \hat{\psi}(\vec{r})\right),
\end{aligned}
$$

satisfies the equation of motion,

$$
\frac{\partial}{\partial t} \widehat{\vec{\Pi}}(\vec{r})=\hat{\vec{\tau}} \Pi(\vec{r})+\hat{\vec{E}}(\vec{r}) \hat{\rho}(\vec{r})+\frac{1}{c} \hat{\vec{j}}(\vec{r}) \times \hat{\vec{B}}(\vec{r})
$$

Aside from the Lorentz force density operator, the relativistic stress vector density operator or the tension density operator $\hat{\vec{\tau}}^{\Pi}(\vec{r})$ is given as the divergence of the stress tensor density operator $\hat{\vec{\tau}} \Pi(\vec{r})$ as follows:

$$
\hat{\tau}^{\Pi k}(\vec{r})=\partial_{l} \hat{\tau}^{\Pi k l}(\vec{r}),
$$

where

$$
\begin{aligned}
\hat{\tau}^{\Pi k}(\vec{r})= & \frac{i \hbar c}{2}\left[\hat{D}_{l}^{+}(\vec{r}) \hat{\bar{\psi}}(\vec{r}) \gamma^{l} \cdot \hat{D}_{k}(\vec{r}) \hat{\psi}(\vec{r})\right. \\
& +\hat{\bar{\psi}}(\vec{r}) \gamma^{l} \hat{D}_{k}(\vec{r}) \hat{D}_{l}(\vec{r}) \hat{\psi}(\vec{r}) \\
& -\hat{D}_{k}^{+}(\vec{r}) \hat{D}_{l}^{+}(\vec{r}) \hat{\bar{\psi}}(\vec{r}) \gamma^{l} \cdot \hat{\psi}(\vec{r}) \\
& \left.-\hat{D}_{k}^{+}(\vec{r}) \hat{\bar{\psi}}(\vec{r}) \gamma^{l} \cdot \hat{D}_{l}(\vec{r}) \hat{\psi}(\vec{r})\right]-\frac{1}{c}(\hat{\vec{j}}(\vec{r}) \times \vec{B}(\vec{r}))^{k},
\end{aligned}
$$

and

$$
\begin{aligned}
\hat{\tau}^{\Pi k l}(\vec{r})= & \frac{i \hbar c}{2}\left[\hat{\bar{\psi}}(\vec{r}) \gamma^{l} \hat{D}_{k}(\vec{r}) \hat{\psi}(\vec{r})\right. \\
& \left.-\hat{D}_{k}^{+}(\vec{r}) \hat{\bar{\psi}}(\vec{r}) \gamma^{l} \cdot \hat{\psi}(\vec{r})\right] .
\end{aligned}
$$

The electronic mass density operator $\hat{M}(\vec{r})$ is further decomposed into the rest mass density operator $-m c^{2}[\hat{\rho}(\vec{r}) / e]$, the kinetic energy density operator $\hat{T}_{M}(\vec{r})$, and the residue $\delta \hat{M}(\vec{r})$ as follows:

$$
\begin{aligned}
& \hat{M}(\vec{r})=-m c^{2} \frac{\hat{\rho}(\vec{r})}{e}+\hat{T}_{M}(\vec{r})+\delta \hat{M}(\vec{r}), \\
& \hat{T}_{M}(\vec{r})=-\frac{\hbar^{2}}{2 m} \cdot \frac{1}{2}\left(\hat{\psi}^{+}(\vec{r}) \hat{D}_{k}^{2}(\vec{r}) \hat{\psi}(\vec{r})\right. \\
&\left.+\hat{D}_{k}^{+2}(\vec{r}) \hat{\psi}^{+}(\vec{r}) \cdot \hat{\psi}(\vec{r})\right), \\
& \delta \hat{M}(\vec{r})=\hat{M}(\vec{r})+m c^{2} \frac{\hat{\rho}(\vec{r})}{e}-\hat{T}_{M}(\vec{r}) .
\end{aligned}
$$

By taking the ensemble average, we arrive at the relativistic extension of the densities. For example, the electronic charge density $\rho(\vec{r})$ is given as

$$
\rho(\vec{r})=\langle\hat{\rho}(\vec{r})\rangle .
$$

It should be noted that the choice of the vacuum and state vectors and the ensemble itself should of course reflect the proper physics of the system we treat. Then, we get

$$
\begin{aligned}
& n_{E_{\text {Dirac }}}(\vec{r})=\left\langle\hat{H}_{\text {Dirac }}(\vec{r})\right\rangle, \\
& n_{T_{M}}(\vec{r})=\left\langle\hat{T}_{M}(\vec{r})\right\rangle, \\
& n_{-e \Phi}(\vec{r})=\langle-e \hat{\Phi}(\vec{r})\rangle, \\
& n_{\delta M}(\vec{r})=\langle\delta \hat{M}(\vec{r})\rangle .
\end{aligned}
$$

And the sum rule of the densities follows

$$
n_{E_{\text {Dirac }}}(\vec{r})=-m c^{2} \frac{\rho(\vec{r})}{e}+n_{T_{M}}(\vec{r})+n_{-e \Phi}(\vec{r})+n_{\delta M}(\vec{r}),
$$

and their integrals with obvious notation: 


$$
E_{\text {Dirac }}=m c^{2} N+T_{M}-e \Phi+\delta M .
$$

The regional decomposition is in order where we observe the relativistic extension of the characteristic regions,

$$
\begin{aligned}
& R_{D_{\text {Dirac }}}=\left\{\vec{r} \mid n_{T_{M}}(\vec{r})>0\right\}, \quad R_{A_{\text {Dirac }}}=\left\{\vec{r} \mid n_{T_{M}}(\vec{r})<0\right\}, \\
& S_{\text {Dirac }}=\left\{\vec{r} \mid n_{T_{M}}(\vec{r})=0\right\} .
\end{aligned}
$$

Regional energy decomposition ${ }^{7-9}$ is found to be the integration over region $R$ of space as in the nonrelativistic case.

It should be noted that if we first switch to the nonrelativistic treatment and second treat the electromagnetic field as the classical radiation field, then the reduction of the kinetic energy density $n_{T_{M}}(\vec{r})$ to the nonrelativistic $n_{T}(\vec{r})$ associated with $n_{-e \Phi}(\vec{r})$ to $n_{V}(\vec{r})$ are dictated as follows:

$$
n_{T_{M}}(\vec{r}) \rightarrow n_{T}(\vec{r}), \quad n_{-e \Phi}(\vec{r}) \rightarrow n_{V}(\vec{r}),
$$

accompanying the reduction of the electronic charge density to the electron density:

$$
\rho(\vec{r}) \rightarrow-e n(\vec{r}) .
$$

\section{Regional work function and regional electric potential}

We shall treat in this section the nonrelativistic grand canonical ensemble without radiation field, ${ }^{12}$ where the density matrix and therefore in particular the electronic energy density $n_{E}(\vec{r})$ are all proved to be given as unique functional of $n(\vec{r}) .^{7-9,12}$

The regional electronic energy $E_{R}$ is the electronic energy of a certain region $R$ in space. The other regions act as spectator or "medium" for the region $R$. This nature is true for extension to the finite temperature case without radiation field using the grand canonical ensemble where all the relevant quantities such as $E_{R}, n(\vec{r})$, or $n_{E}(\vec{r})$ are defined by the thermodynamic quantities. ${ }^{7}$ The manipulation of electron is then treated as a thermodynamic process and again the relevant quantities are all given as the unique functional of $n(\vec{r}) .^{7,12}$

For example, removal of the electron from the system to the reservoir gives Gibbs chemical potential $\mu_{G}$. Using Gibbs grand canonical ensemble, we arrive at the expression of the Gibbs chemical potential $\mu_{G}$ as follows: ${ }^{7}$

$$
\mu_{G}=\mu_{R}+\sum_{R^{\prime}(\neq R)} \alpha_{R^{\prime} R},
$$

where

$$
\begin{aligned}
& \mu_{R}=\left(\frac{\partial E_{R}}{\partial N_{R}}\right)_{S, v, N_{R^{\prime}(\neq R)}}, \\
& \alpha_{R^{\prime} R}=\left(\frac{\partial E_{R^{\prime}}}{\partial N_{R}}\right)_{S, v, N_{R^{\prime}(\neq R)}} .
\end{aligned}
$$

The regional chemical potential $\mu_{R}$ refers to the regional contribution to the $\mu_{G}$. If an electron is withdrawn from a region $R$ and reaches the reservoir, the regional electronic energy $E_{R}$ changes according to Eq. (58) and the $\mu_{R}$ gives the energy change per one electron. On the other hand, the passage of the electron through the system to the reservoir should inevitably influence the electronic energies of the other regions $R$ 's, where the regional electronic energy $E_{R^{\prime}}$ changes according as Eq. (59) and the $\alpha_{R^{\prime} R}$ gives the energy change per one electron. If an electron is withdrawn from a region $R$ and passes through another region $R^{\prime}$, then the electron is treated here external to the region $R^{\prime}$. Namely, the $R^{\prime}$ here acts as a spectator to the passing electron and therefore the energy change in $E_{R^{\prime}}$ as measured by $\alpha_{R^{\prime} R}$ in Eq. (59) should then be "electrostatic" in nature. This should be the electric potential first observed by Volta as proved by Herring and Nichols. ${ }^{17}$ It should be noted that manipulation of electron solely in the particular region leads to the thermodynamic definition of work function. ${ }^{17}$ As demonstrated by Volta for a pair of regions $R$ and $R^{\prime}$ in contact with each other, the contact potential difference is the difference in the regional work function as proved by Herring and Nichols: ${ }^{17}$

$$
\phi_{R}-\phi_{R^{\prime}}=\Phi_{R^{\prime}}-\Phi_{R}
$$

where $\phi_{R}$ denotes the Volta electric potential for the region $R$ and $\Phi_{R}$ denotes the work function of the region $R$. This is the consequence of the chemical equilibrium in between a pair of regions in contact with each other: ${ }^{17}$

$$
\mu_{G}=-e \Phi_{R}-e \phi_{R}=-e \Phi_{R^{\prime}}-e \phi_{R^{\prime}},
$$

where the Gibbs chemical potential $\mu_{G}$ is constant from region to region in contact with each other under the condition of global chemical equilibrium.

On the other hand, the electrostatic effect is long-ranged. As a matter of fact, the electron is negatively charged and has non-negligible interaction even for spectator region $R^{\prime \prime}$ not directly in contact with the region $R$. For example, the surface dipole of the spectator region $R^{\prime}$ can contribute to the long-ranged electrostatic interaction with the electron. Hence, in our theory, the intrinsic Volta electric potential $\phi_{R}$ for the region $R$ is expressed by the sum of $\alpha_{R^{\prime} R}$ over the spectator complementary regions $R^{\prime}$ to $R$ :

$$
-e \phi_{R}=\sum_{R^{\prime}(\neq R)} \alpha_{R^{\prime} R}
$$

Therefore, substituting Eq. (62) into Eq. (61) and comparing it with Eq. (57), we arrive at the intrinsic Herring-Nichols work function $\Phi_{R}$ for the region $R$ as follows:

$$
-e \Phi_{R}=\mu_{R}
$$

Thus, the thermodynamic extension of the electronic energy density $n_{E}(\vec{r})$ turned out to be observable in electrochemistry: in terms of the intrinsic Volta electric potential $\phi_{R}$ and the intrinsic Herring-Nichols work function $\Phi_{R}$. Even for the same crystal, the $\phi_{R}$ and the $\Phi_{R}$ are dependent on surface morphologies or crystallographic orientations, while the sum of these gives the constant value $\mu_{G}$ for the same crystal according to Eq. (61). ${ }^{17,18}$ 


$$
\mathrm{He}+\mathrm{H}^{+} \rightarrow \mathrm{HeH}^{+}
$$

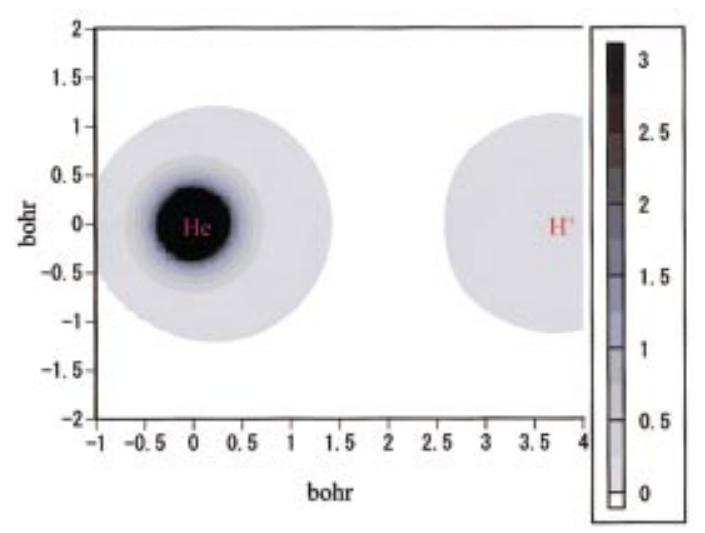

(a)

$$
\mathrm{He}+\mathrm{H}^{+} \rightarrow \mathrm{HeH}^{+}
$$

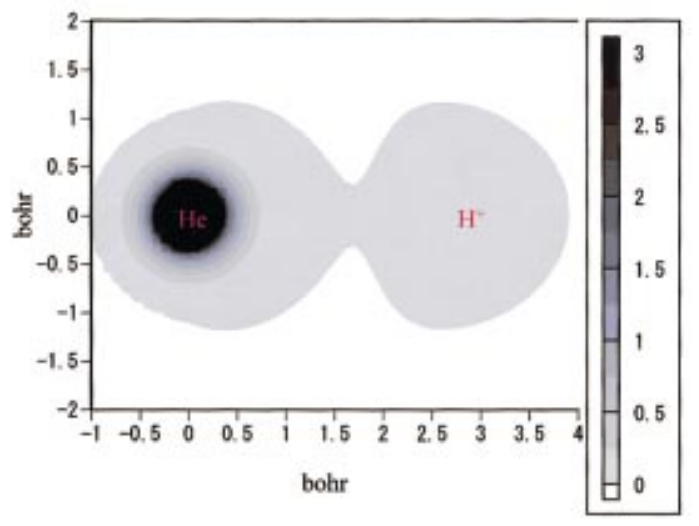

(b)
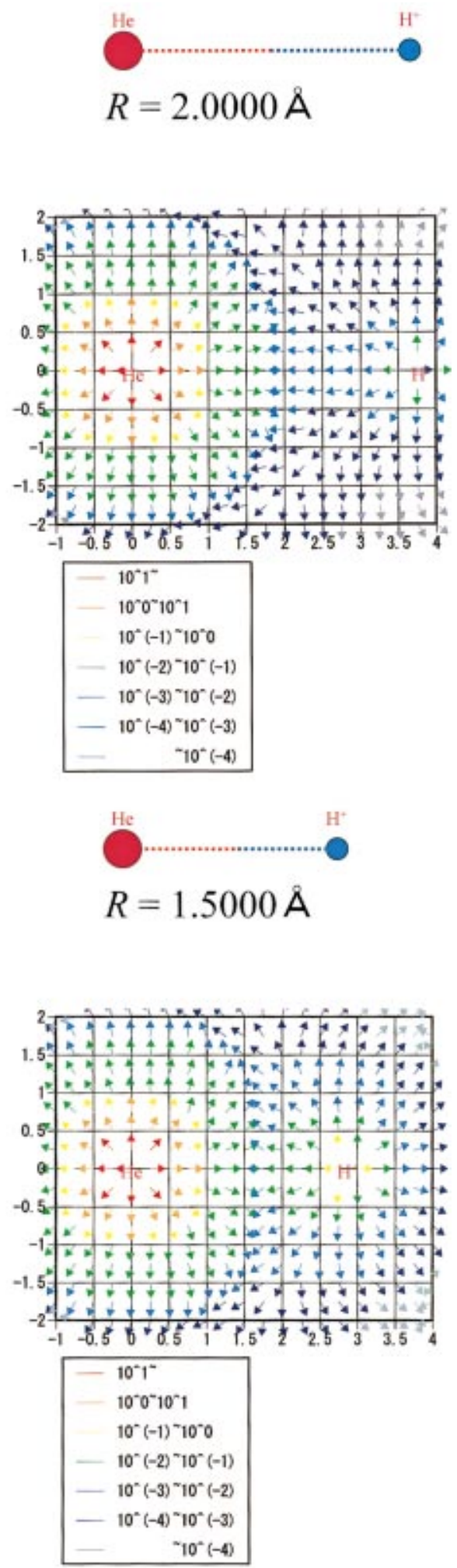

FIG. 1. (Color) Characteristic densities are demonstrated for a protonation reaction to He. The reaction coordinate is the internucleus distance $R$ $=r\left(\mathrm{He}-\mathrm{H}^{+}\right)$. The equilibrium distance is $r\left(\mathrm{He}-\mathrm{H}^{+}\right)=0.7735 \AA$. The maps of $n_{T}(\vec{r})$ and $\vec{\tau}^{S}(\vec{r})$ are shown in $\left(\right.$ a) at $r\left(\mathrm{He}-\mathrm{H}^{+}\right)=2.0 \AA$ and $(\mathrm{b})$ at $r\left(\mathrm{He}-\mathrm{H}^{+}\right)=1.5 \AA$. The left-hand side is the map of $n_{T}(\vec{r})$ and the right-hand side $\vec{\tau}(\vec{r})$. The scale of length is a.u. and the scale of density is hartree bohr ${ }^{-3}$. For $\vec{\tau}^{S}(\vec{r})$, the arrow shows the direction of the vector while the color gradation shows the strength gradation. The maps of the external potential energy density $n_{V}(\vec{r})$, the interelectron potential energy density $n_{W}(\vec{r})$, and the total energy density $n_{E}(\vec{r})$ are shown in $(\mathrm{c})$ at $r\left(\mathrm{He}-\mathrm{H}^{+}\right)=2.0 \AA$ and $(\mathrm{d})$ at $r\left(\mathrm{He}-\mathrm{H}^{+}\right)=1.5 \AA$. The upper left-hand side is the map of $n_{V}(\vec{r})$ and the upper right-hand side $n_{W}(\vec{r})$, while the lower side $n_{E}(\vec{r})$. 


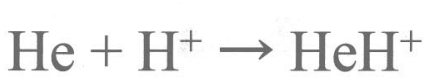

$R=2.0000 \AA$
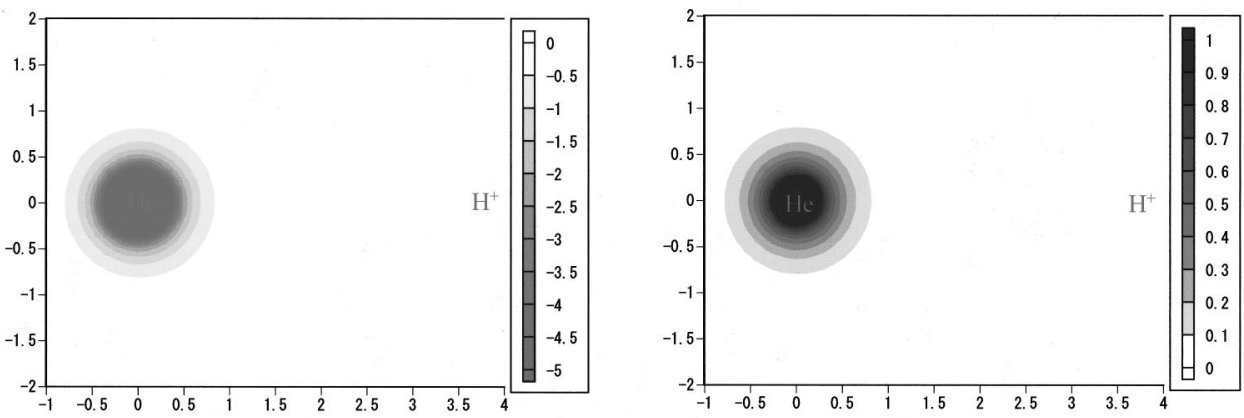

(c)
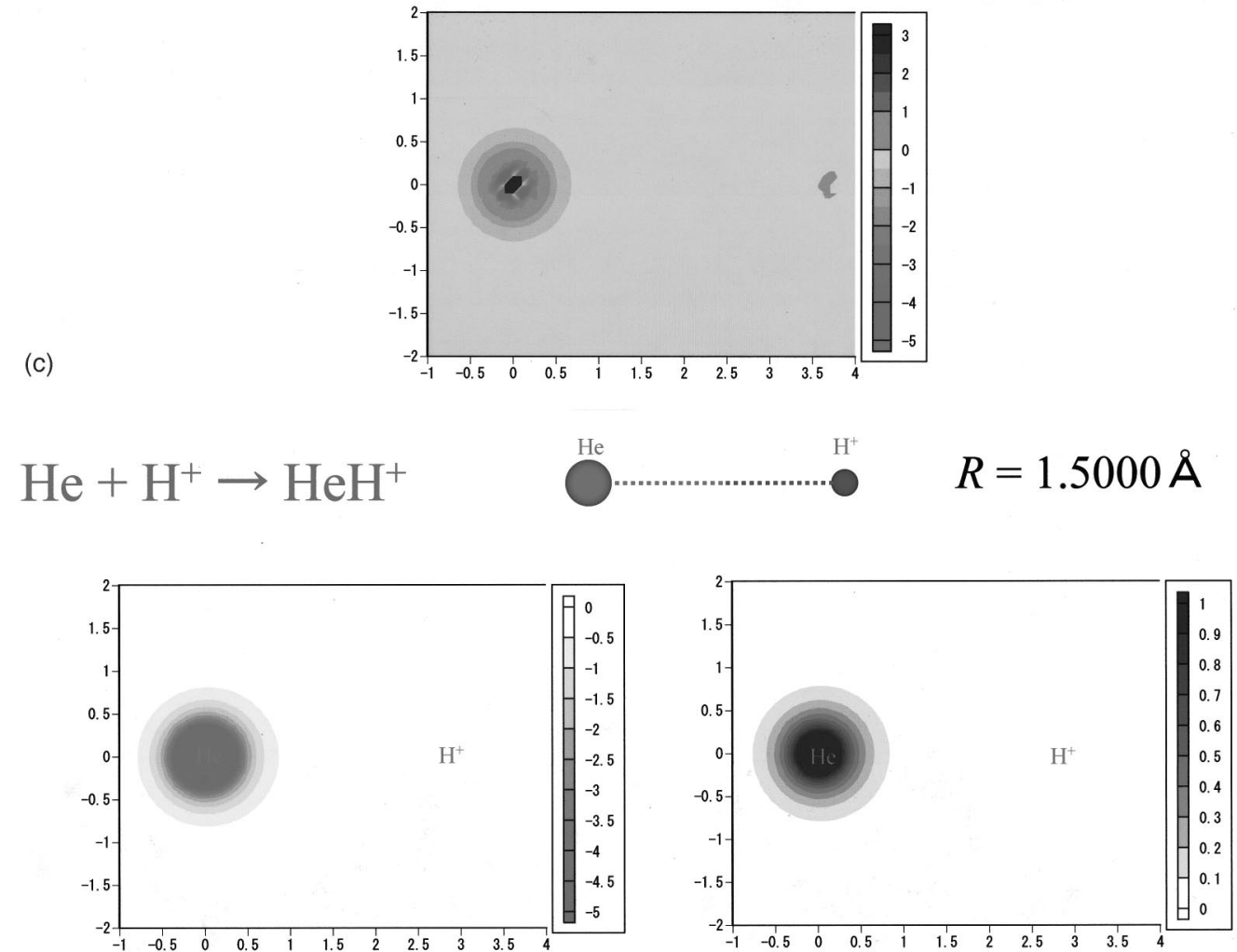

(d)

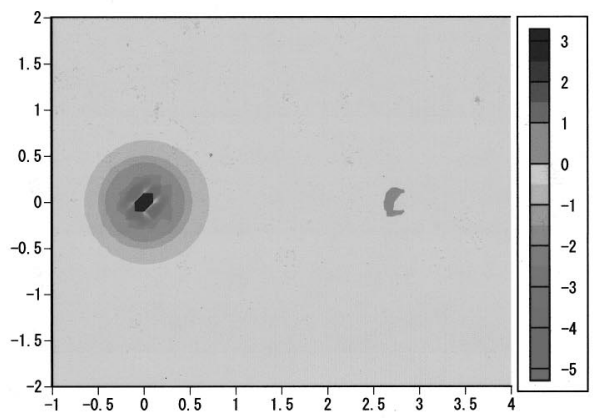

FIG. 1. (Continued.)

\section{APPLICATION}

\section{A. $\mathrm{H}$ atom}

Here we pick up first a hydrogen-like atom without radiation field for which we have an analytical exact expression of the relativistic kinetic energy density $n_{T_{M}}(\vec{r})$ :

$$
n_{T_{M}}(\vec{r})=-\left(E_{\text {Dirac }}-m c^{2}\right) \frac{\rho(\vec{r})}{e}-n_{-e \Phi}(\vec{r})-n_{\delta M}(\vec{r}),
$$

and its nonrelativistic version $n_{T}(\vec{r})$, 


$$
n_{T}(\vec{r})=(E-\nu(\vec{r})) n(\vec{r}),
$$

with

$$
\nu(\vec{r})=-e \phi(\vec{r})=-\frac{Z e^{2}}{|\vec{r}|},
$$

where $Z$ is the nuclear charge number. These expressions are valid for any excited bound states as well as the ground state.
In the ground state, the nucleus is surrounded by the electronic interface of radius $r_{S_{\text {Dirac }}}$ for the relativistic case and $r_{S}$ for the nonrelativistic case within which the kinetic energy density is positive leaving negative outside:

$$
r_{S_{\text {Dirac }}}=\frac{\hbar^{2}}{Z m e^{2}}\left(\sqrt{1-Z^{2} \alpha^{2}}+\sqrt[4]{1-Z^{2} \alpha^{2}}\right), \quad \alpha=\frac{e^{2}}{\hbar c},
$$

\section{$\mathrm{C}_{2} \mathrm{H}_{4}+\mathrm{HF} \rightarrow \mathrm{CH}_{3} \mathrm{CH}_{2} \mathrm{~F}$}
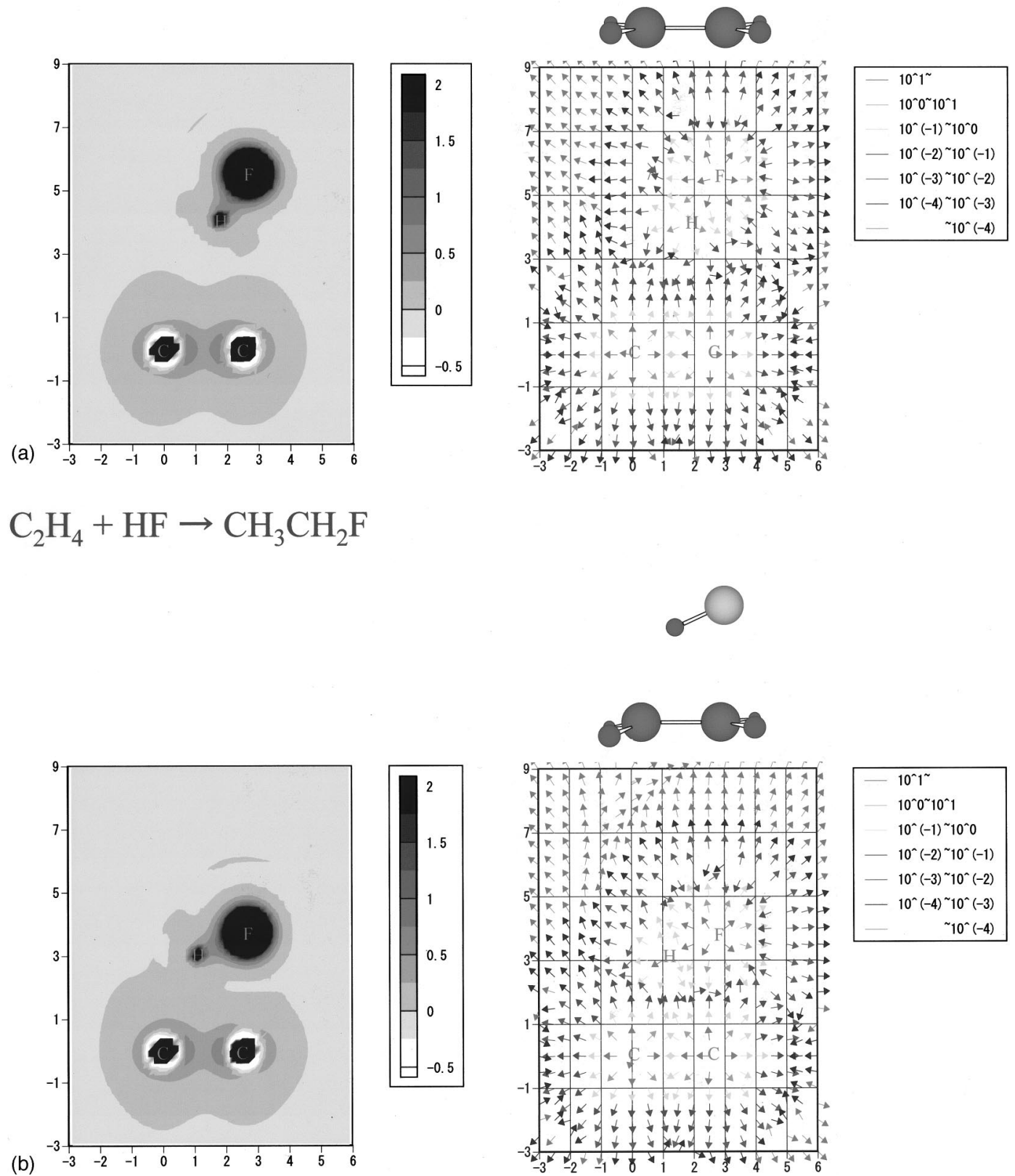

FIG. 2. Characteristic densities are demonstrated for an addition reaction of $\mathrm{HF}$ to $\mathrm{C}_{2} \mathrm{H}_{4}$ along the IRC of $C s$ symmetry in (a)-(e) in which (c) at the transition state (TS) corresponds to the saddle point on the potential energy surface and (e) at the product complex. The left-hand side is the map of $n_{T}(\vec{r})$ and the right-hand side $\vec{\tau}^{S}(\vec{r})$. The scale of length is a.u. and the scale of density is hartree bohr ${ }^{-3}$. For $n_{T}(\vec{r})$ the grey background denotes negative region $R_{A}$. For $\vec{\tau}(\vec{r})$ the arrow shows the direction of the vector while the color gradation shows the strength gradation. 


\section{$\mathrm{C}_{2} \mathrm{H}_{4}+\mathrm{HF} \rightarrow \mathrm{CH}_{3} \mathrm{CH}_{2} \mathrm{~F}$}

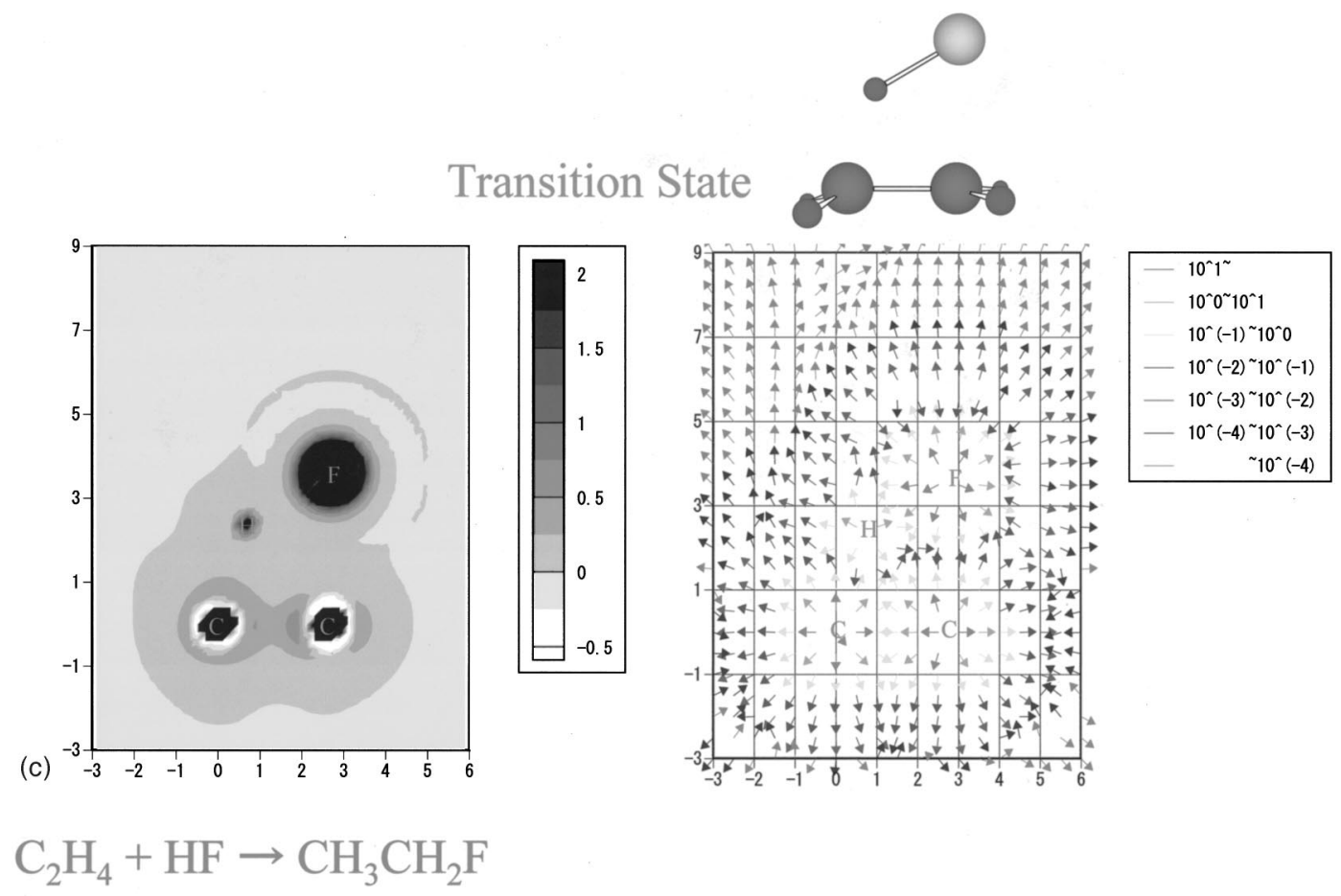

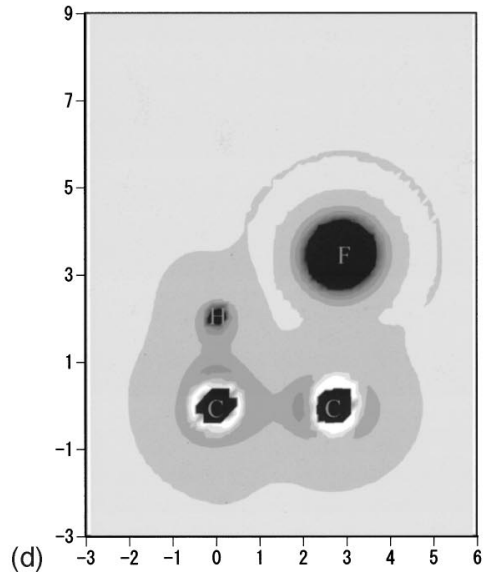

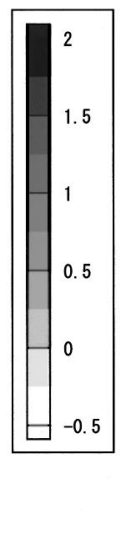

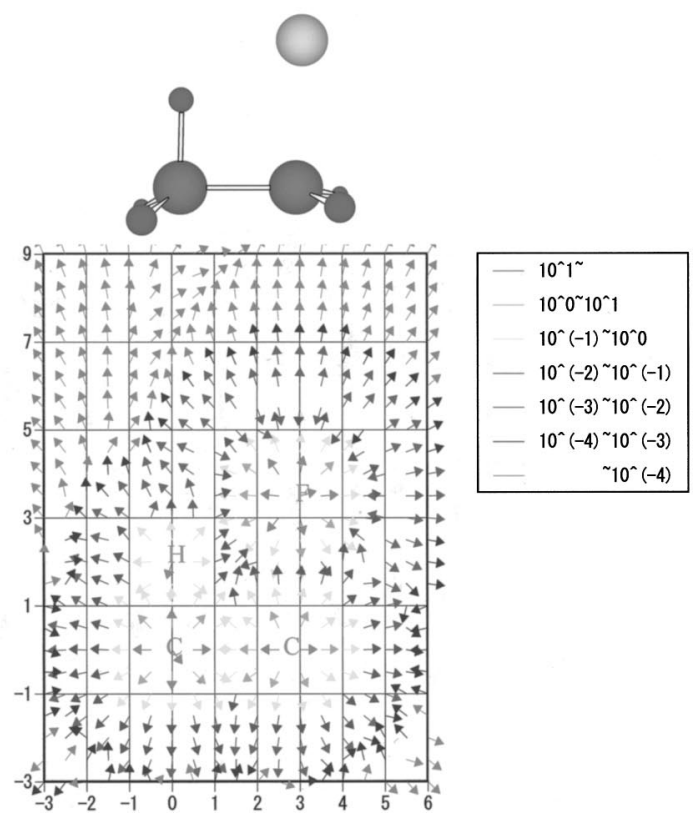

FIG. 2. (Continued.)

$$
r_{S}=\frac{2 \hbar^{2}}{Z m e^{2}},
$$

so that the intrinsic shape is this electronic drop region just surrounded by this electronic interface. The radius $r_{S_{\text {Dirac }}}$ for the relativistic case is smaller than the nonrelativistic value $r_{S}$, which ratio approaches 0 as $Z$ approaches 137 . The intrinsic shape of the $\mathrm{H}$ atom with $Z=1$ has the radius $r_{S_{\text {Dirac }}}$ $\approx r_{S}=2$ a.u. $\approx 1.058 \AA$, which is comparable to the standard atomic radius of $\mathrm{H}$ atom, 1.5 a.u. $\approx 0.79 \AA$, reduced by a factor of $75 \%$.

\section{B. Chemical reaction systems}

We shall apply the nonrelativistic theory to several chemical reaction systems in the electronic ground states 


$$
\mathrm{C}_{2} \mathrm{H}_{4}+\mathrm{HF} \rightarrow \mathrm{CH}_{3} \mathrm{CH}_{2} \mathrm{~F}
$$
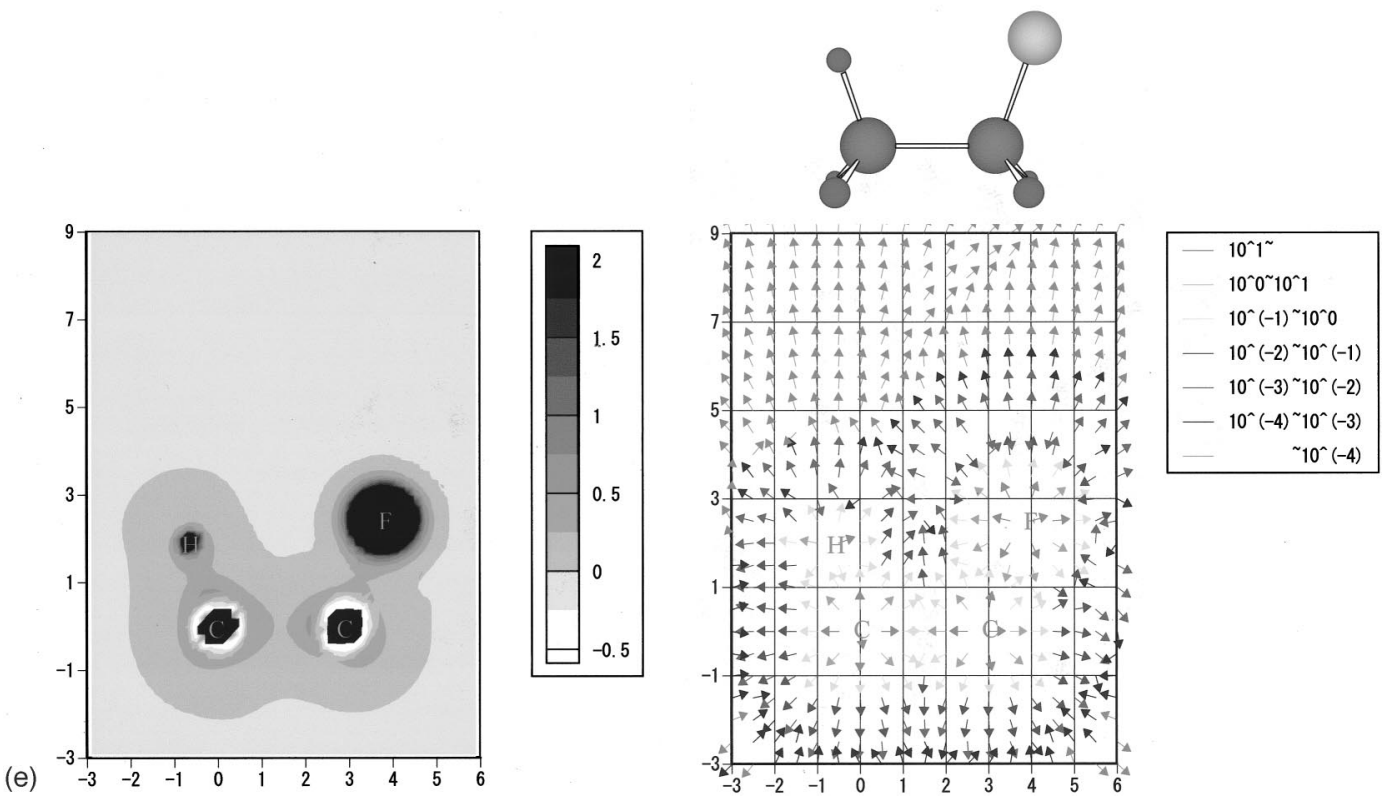

FIG. 2. (Continued.) without radiation field, where no additional external electromagnetic field is present but the electrostatic field of nuclei. Particular interest is put on the kinetic energy density $n_{T}(\vec{r})$ that is reduced from Eqs. (2) and (17) to

$$
n_{T}(\vec{r})=\left\langle-\frac{\hbar^{2}}{2 m} \cdot \frac{1}{2}\left(\hat{\chi}^{+}(\vec{r}) \partial_{k}^{2} \hat{\chi}(\vec{r})+\partial_{k}^{2} \hat{\chi}^{+}(\vec{r}) \cdot \hat{\chi}(\vec{r})\right)\right\rangle .
$$

Since the electronic state is in the stationary state, we conclude from Eqs. (8) and (9) that the tension density $\vec{\tau}^{S}(\vec{r})$ exactly cancels the external force density $\vec{K}(\vec{r})$ :

$$
0=\vec{\tau}^{S}(\vec{r})+\vec{K}(\vec{r}),
$$

where

$$
\begin{aligned}
\tau^{S k}(\vec{r})= & \left\langle\frac { \hbar ^ { 2 } } { 4 m } \left[\hat{\chi}^{+}(\vec{r}) \partial_{k} \partial_{l}^{2} \hat{\chi}(\vec{r})+\partial_{k} \partial_{l}^{2} \hat{\chi}^{+}(\vec{r})\right.\right. \\
& \left.\left.\cdot \hat{\chi}(\vec{r})-\partial_{k} \hat{\chi}^{+}(\vec{r}) \cdot \partial_{l}^{2} \hat{\chi}(\vec{r})-\partial_{l}^{2} \hat{\chi}^{+}(\vec{r}) \cdot \partial_{k} \hat{\chi}(\vec{r})\right]\right\rangle,
\end{aligned}
$$

and

$$
\begin{aligned}
K^{k}(\vec{r})= & \left\langle-\hat{\chi}^{+}(\vec{r})\left(\partial_{k} \nu(\vec{r})\right.\right. \\
& \left.\left.+\int d^{3} \vec{s} \hat{\chi}^{+}(\vec{s}) \partial_{k} w(\vec{r}, \vec{s}) \hat{\chi}(\vec{s})\right) \cdot \hat{\chi}(\vec{r})\right) .
\end{aligned}
$$

We shall here select the following five reaction systems and show the figures of the kinetic energy densities as well as the other energy densities along the intrinsic reaction coordinates (IRCs). ${ }^{1-3}$

\section{Protonation reaction to $\mathrm{He}$}

Here we examine a protonation reaction to He utilizing the basis set given in Ref. 10. The reaction coordinate is the internucleus distance $r\left(\mathrm{He}-\mathrm{H}^{+}\right)$. The reaction energy is exothermic and there appears no transition state down to the equilibrium distance $r\left(\mathrm{He}-\mathrm{H}^{+}\right)=0.7735 \AA$. The maps of the kinetic energy density $n_{T}(\vec{r})$ and the tension density $\vec{\tau} S(\vec{r})$ are shown in Fig. 1(a) at $r\left(\mathrm{He}-\mathrm{H}^{+}\right)=2.0 \AA$ and Fig. 1(b) at $r\left(\mathrm{He}-\mathrm{H}^{+}\right)=1.5 \AA$. At $r\left(\mathrm{He}-\mathrm{H}^{+}\right)=\infty$, the $\mathrm{He}$ atomic nucleus is surrounded by the $S$ of radius $\approx 0.676 \AA$ within which the $n_{T}(\vec{r})$ is positive, so that the $R_{D}$ is this sphere just defined. This intrinsic shape of the He atom is comparable to the standard atomic radius $0.49 \AA$ of $\mathrm{He}$ atom reduced by a factor of $\approx 72 \%$; it should be noted that the smaller shape as compared with the $\mathrm{H}$ atom is well reproduced together with a similar value of the reduction factor.

At $r\left(\mathrm{He}-\mathrm{H}^{+}\right)=2.0 \AA$, we observe two disjoint $R_{D}$ 's, one for $\mathrm{He}$ and the other for $\mathrm{H}^{+}$. As reaction proceeds, the kinetic energy density $n_{T}(\vec{r})$ becomes gradually polarized toward the internucleus region. The polarization for the $R_{D}$ is found to be most drastic and then the $S$ dictates the drastic change of the shape of the $\mathrm{He}-\mathrm{H}^{+}$intermediate structure. Actually, the $R_{D}$ for $\mathrm{He}$ extends to the $R_{D}$ for $\mathrm{H}^{+}$at $r\left(\mathrm{He}-\mathrm{H}^{+}\right)=1.5 \AA$. Namely, the two disjoint regions of the electronic drops, $R_{D}$ for $\mathrm{He}$ and $R_{D}$ for $\mathrm{H}^{+}$, have been fused there. Although there is found no saddle point on the potential energy curve along the reaction coordinate, we may identify the intrinsic electronic transition state to the coalescent point. Very swift change of the delocalized $R_{D}$ of valence electrons thus dictates the transformation (formationscission) of chemical bonds along the reaction coordinate. Furthermore, along the course of chemical reaction, we ob- 
serve a pair of sources of tension located at each nucleus of $\mathrm{He}$ and $\mathrm{H}^{+}$. The two tension fields collide and demonstrate a very interesting figure where the tension flow traced by the tension density $\vec{\tau} \vec{S}(\vec{r})$ defines the catastrophe point in between the two nuclei. The catastrophe point of the tension flow moves toward the $\mathrm{He}$ nucleus as reaction proceeds when the tension density for the side of the $\mathrm{H}^{+}$nucleus gets strengthened. It should be noted that the electronic state is in the stationary state, and therefore the tension density $\vec{\tau} S(\vec{r})$ exactly cancels the external force density $\vec{K}(\vec{r})$ as shown in Eq. (70). Then, if we reverse the direction of the arrow, then we get the map of the external force density $\vec{K}(\vec{r})$ without calculating the interelectron integral in Eq. (72). Thus we can visualize both the tension and the external force exerted on the electron using the same figure simultaneously.

The maps of the external potential energy density $n_{V}(\vec{r})$, the interelectron potential energy density $n_{W}(\vec{r})$, and the to

$$
\mathrm{C}_{2} \mathrm{H}_{2}+\mathrm{HF} \rightarrow \mathrm{CH}_{2}=\mathrm{CHF}
$$
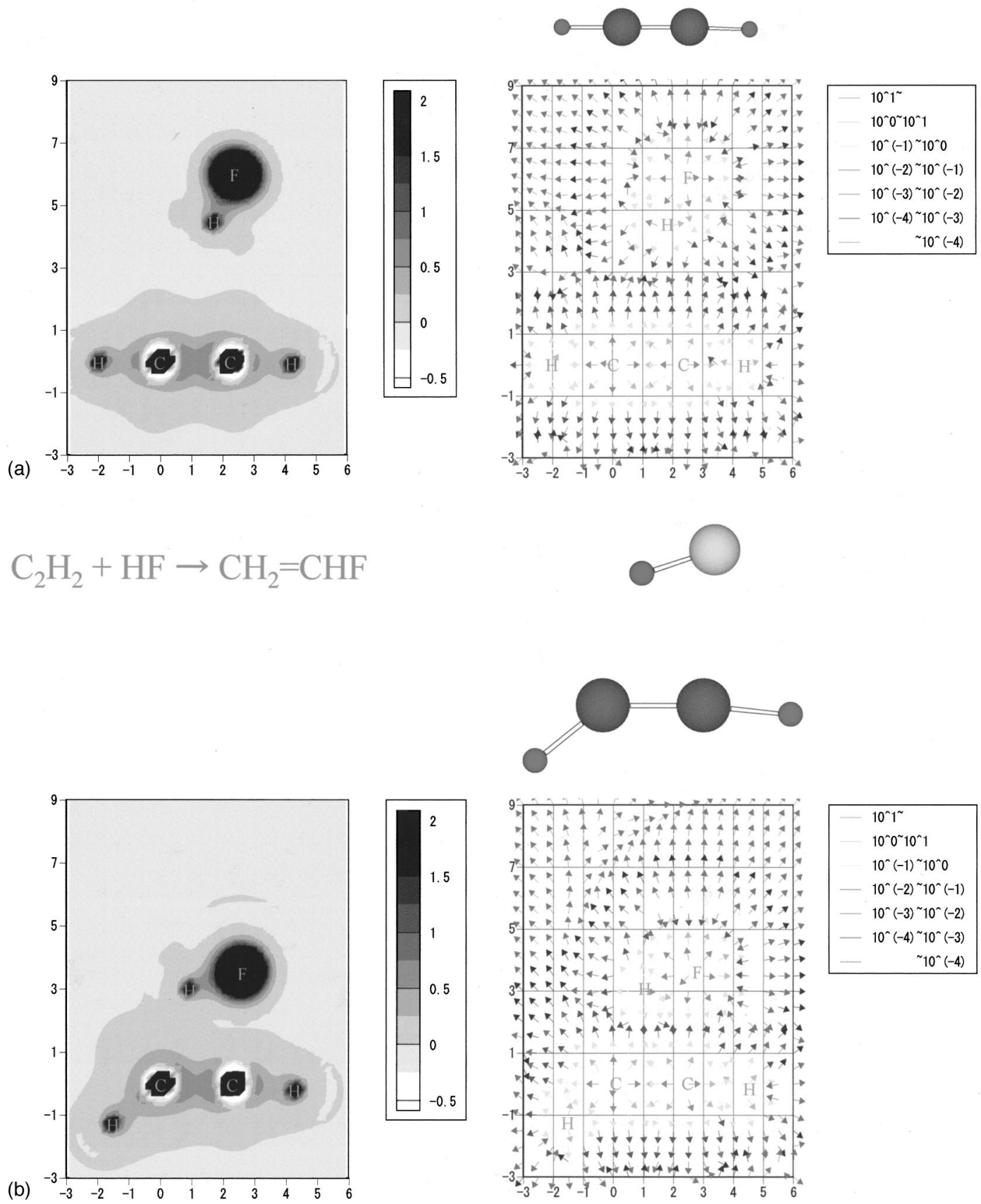

FIG. 3. Characteristic densities are demonstrated for an addition reaction of $\mathrm{HF}$ to $\mathrm{C}_{2} \mathrm{H}_{2}$ along the IRC. For other details, see the caption of Fig. 2. 


\section{$\mathrm{C}_{2} \mathrm{H}_{2}+\mathrm{HF} \rightarrow \mathrm{CH}_{2}=\mathrm{CHF}$}
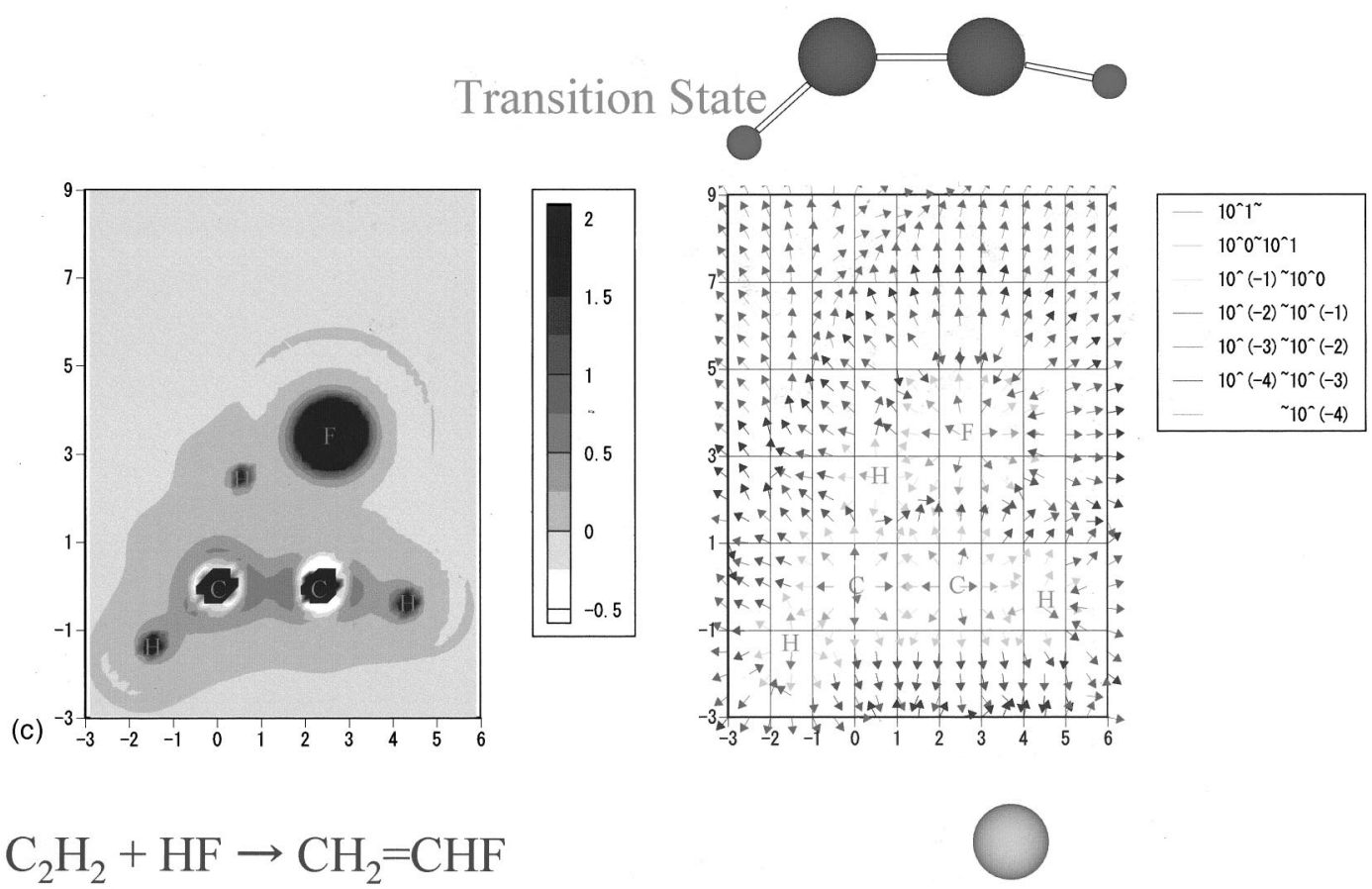

$\mathrm{C}_{2} \mathrm{H}_{2}+\mathrm{HF} \rightarrow \mathrm{CH}_{2}=\mathrm{CHF}$

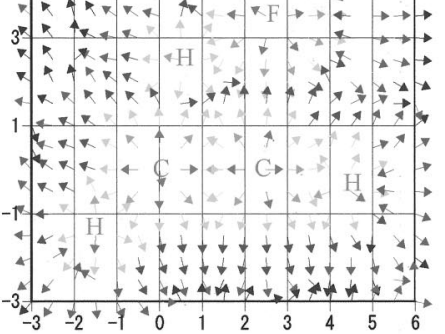

$90(-4)$
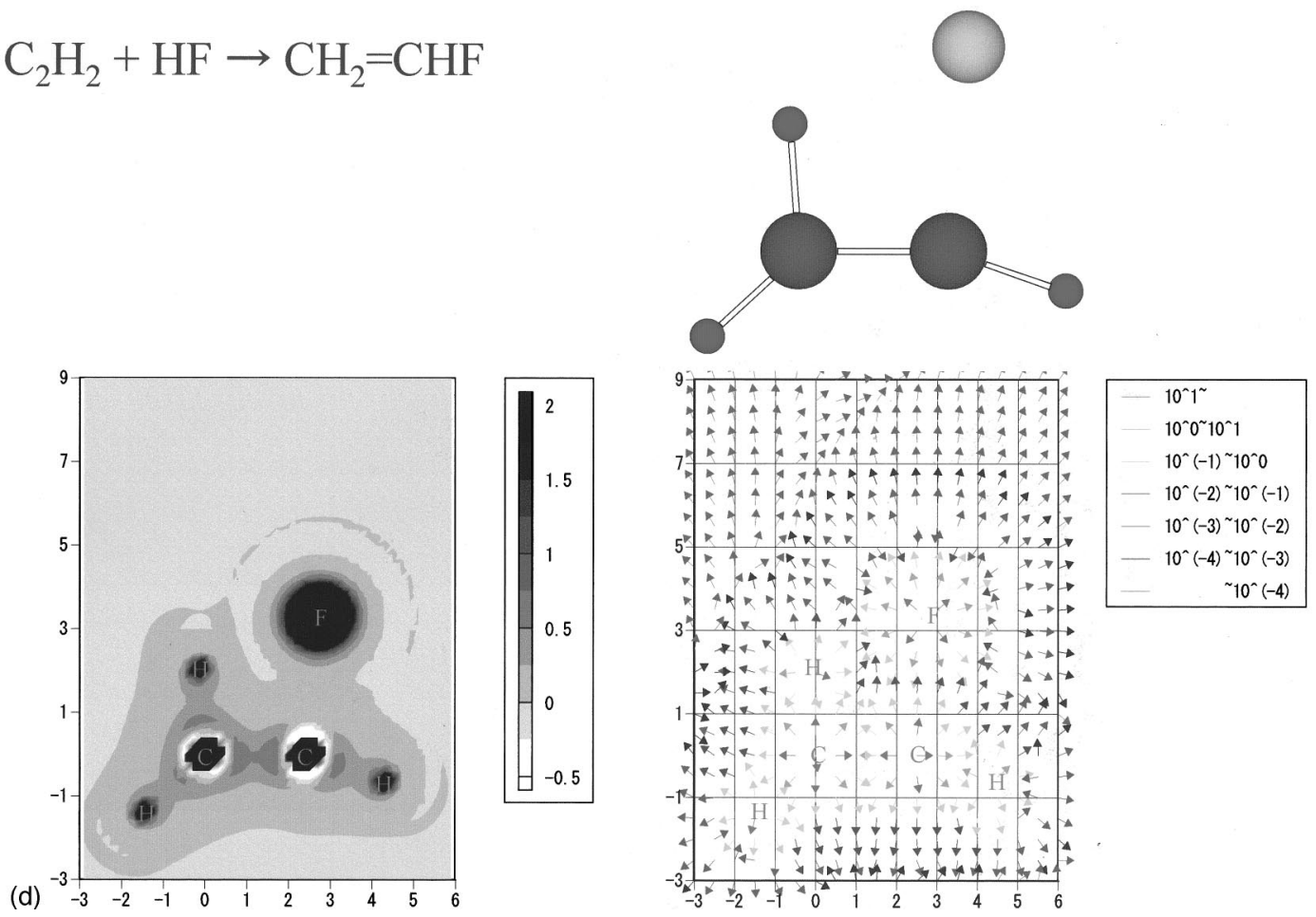

FIG. 3. (Continued.)

tal energy density $n_{E}(\vec{r})$ are shown in Fig. 1(c) at $r\left(\mathrm{He}-\mathrm{H}^{+}\right)=2.0 \AA$ and Fig. $1(\mathrm{~d})$ at $r\left(\mathrm{He}-\mathrm{H}^{+}\right)=1.5 \AA$. The upper left-hand side is the map of $n_{V}(\vec{r})$ and the upper righthand side $n_{W}(\vec{r})$, while the lower side $n_{E}(\vec{r})$. The external potential energy density $n_{V}(\vec{r})$ is negative definite while $n_{W}(\vec{r})$ positive definite, and together with $n_{E}(\vec{r})$ they all do not show, in contrast to $n_{T}(\vec{r})$ and $\vec{\tau}^{S}(\vec{r})$, very characteristic change of the delocalized valence electrons that dictates the transformation (formation scission) of chemical bonds along the reaction coordinate.

Therefore, we shall focus on $n_{T}(\vec{r})$ and $\vec{\tau}^{S}(\vec{r})$ exclusively in the following reaction systems.

\section{Addition reaction of $\mathrm{HF}$ to $\mathrm{C}_{2} \mathrm{H}_{4}$}

Here we examine an addition reaction of $\mathrm{HF}$ to $\mathrm{C}_{2} \mathrm{H}_{4}$. The maps of the kinetic energy density $n_{T}(\vec{r})$ and the tension 


$$
\mathrm{C}_{2} \mathrm{H}_{2}+\mathrm{HF} \rightarrow \mathrm{CH}_{2}=\mathrm{CHF}
$$
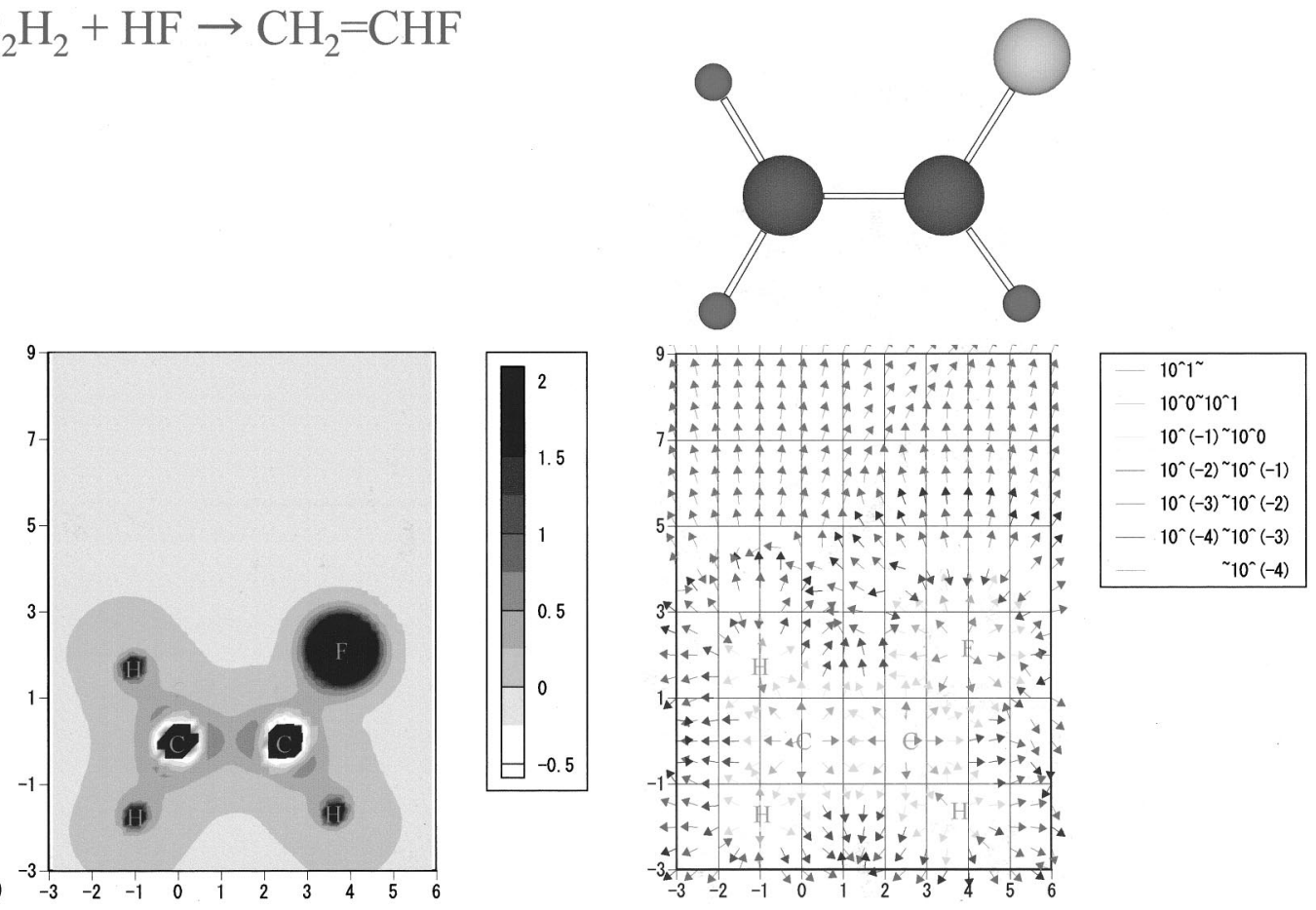

FIG. 3. (Continued.)

density $\vec{\tau}^{S}(\vec{r})$ are shown along the IRC of $C s$ symmetry in Figs. 2(a)-2(e) in which (c) at the transition state (TS) corresponding to the saddle point on the potential energy surface and (e) at the product complex. In the reactant cell of the reaction system (a), we observe two disjoint $R_{D}$ 's, one for $\mathrm{C}_{2} \mathrm{H}_{4}$ and the other for HF. A pair of disjoint $R_{A}$ 's around the innermost $R_{D}$ 's of C's may represent the boundaries of $K$ and $L$ shells discriminating heavily localized core electrons adhered to nuclei and valence electrons that constitute the localized $\sigma$ bond and the delocalized $\pi$ bond in $\mathrm{C}_{2} \mathrm{H}_{4}$. Also found in HF the characteristic $R_{D}$ that shows the behavior of the donation of the occupied $\sigma$ electron of $\mathrm{H}$ to $\mathrm{F}$ and the backdonation of $\pi$ electron of $\mathrm{F}$ to the vacant $\pi$ orbital of $\mathrm{H}$. As reaction proceeds, (b), the kinetic energy density $n_{T}(\vec{r})$ becomes gradually polarized toward the intermolecule region and merges at the corner of $\mathrm{H}$. This coalescence dictates the electronic transition state that supercedes the saddle point on the potential energy surface. At the transition state, (c), the two disjoint $R_{D}$ 's of the reactant molecules are completely fused also at the corner of $\mathrm{F}$. The scission of the $\mathrm{H}-\mathrm{F}$ bond starts at (d) and ends at (e) where the newly completed $\mathrm{H}-\mathrm{C}$ and $\mathrm{F}-\mathrm{C}$ bonds are contrasted with the weakened $\mathrm{C}-\mathrm{C}$ bond in terms of the change in the magnitude of the $n_{T}(\vec{r})$ and the associated characteristic change of the $\vec{\tau}^{S}(\vec{r})$.

\section{Addition reaction of $\mathrm{HF}$ to $\mathrm{C}_{2} \mathrm{H}_{2}$}

Here we examine an addition reaction of $\mathrm{HF}$ to $\mathrm{C}_{2} \mathrm{H}_{2}$. The maps of the kinetic energy density $n_{T}(\vec{r})$ and the tension density $\vec{\tau} S(\vec{r})$ are shown along the IRC of $C s$ symmetry in Figs. 3(a)-3(e) in which Fig. 3(c) at the transition state (TS) corresponding to the saddle point on the potential energy surface and (e) at the product complex. A qualitatively similar feature to the preceding reaction system is anticipated and actually found, except the change of the $\mathrm{C}-\mathrm{C}$ triple bond to double bond keeping the stronger magnitude of the $n_{T}(\vec{r})$ along the IRC as compared with the change of the $\mathrm{C}-\mathrm{C}$ double bond to single bond in the former.

\section{Hydrogen abstraction reaction of $\mathrm{NH}_{3}^{+}$from $\mathrm{HF}$}

Here we examine a hydrogen abstraction reaction of $\mathrm{NH}_{3}^{+}$from HF. ${ }^{3,9,19}$ The maps of the kinetic energy density $n_{T}(\vec{r})$ and the tension density $\vec{\tau}^{S}(\vec{r})$ are shown along the IRC of $C s$ symmetry in Figs. 4(a)-4(e) in which (c) at the transition state (TS) corresponding to the saddle point on the potential energy surface and (e) at the product complex. In the reactant cell of the reaction system, (a), we observe two disjoint $R_{D}$ 's, one for $\mathrm{NH}_{3}^{+}$and the other for HF. A disjoint $R_{A}$ around the innermost $R_{D}$ of $\mathrm{N}$ may represent the boundary of $K$ and $L$ shells discriminating heavily localized core electrons adhered to nucleus and valence electrons that constitute the localized $\sigma$ bond to $\mathrm{H}$. As reaction proceeds, (b), the kinetic energy density $n_{T}(\vec{r})$ becomes gradually polarized toward the intermolecule region and merges at the corner of $\mathrm{H}$. This coalescence dictates the electronic transition state that supercedes the saddle point on the potential energy surface. At the transition state, (c), the scission of $\mathrm{H}-\mathrm{F}$ bond starts and then proceeds through (d) and ends at (e) where the newly completed $\mathrm{H}-\mathrm{N}$ bond is contrasted with the broken $\mathrm{H}-\mathrm{F}$ bond in terms of the change in the magnitude of the $n_{T}(\vec{r})$ and the associated characteristic change of the $\vec{\tau} S(\vec{r})$. Also at the product complex, (e), the releasing $\mathrm{F}$ is slightly bound to $\mathrm{NH}_{4}^{+}$and a trace of $R_{A}$ around the innermost $R_{D}$ of F may represent the discrimination of $K$ and $L$ shells in F. 


\section{Hydrogen abstraction reaction of $\mathrm{NH}_{3}^{+}$from $\mathrm{NH}_{3}$}

Here we examine a hydrogen abstraction reaction of $\mathrm{NH}_{3}^{+}$from $\mathrm{NH}_{3} \cdot{ }^{3,9,19}$ The maps of the kinetic energy density $n_{T}(\vec{r})$ and the tension density $\vec{\tau} S(\vec{r})$ are shown along the IRC

$$
\mathrm{NH}_{3}{ }^{+}+\mathrm{HF} \rightarrow \mathrm{NH}_{4}^{+}+\mathrm{F}
$$

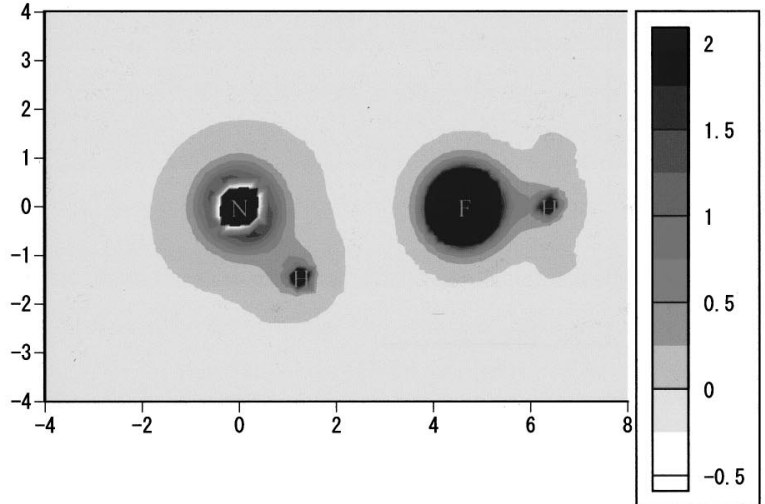

(a)

$$
\mathrm{NH}_{3}^{+}+\mathrm{HF} \rightarrow \mathrm{NH}_{4}^{+}+\mathrm{F}
$$

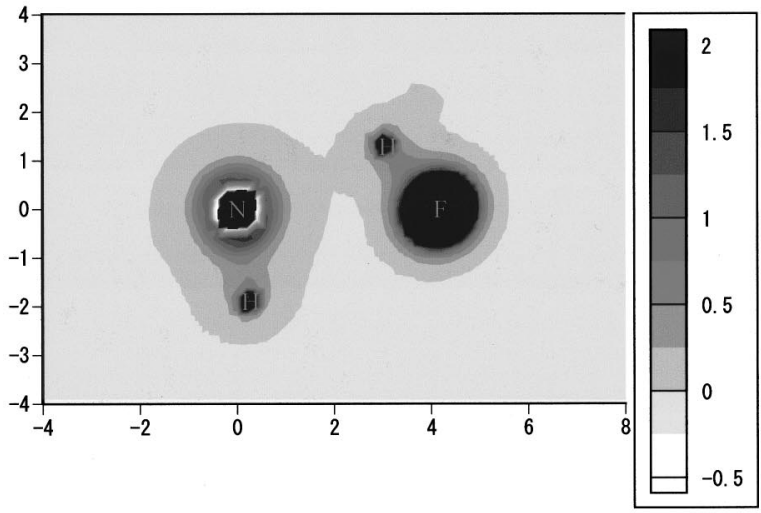

(b) of $C s$ symmetry in Figs. 5(a)-5(e) in which (a) at the reactant complex, (c) at the transition state (TS) corresponding to the saddle point on the potential energy surface and (e) at the product complex. Qualitatively similar feature to the preced-
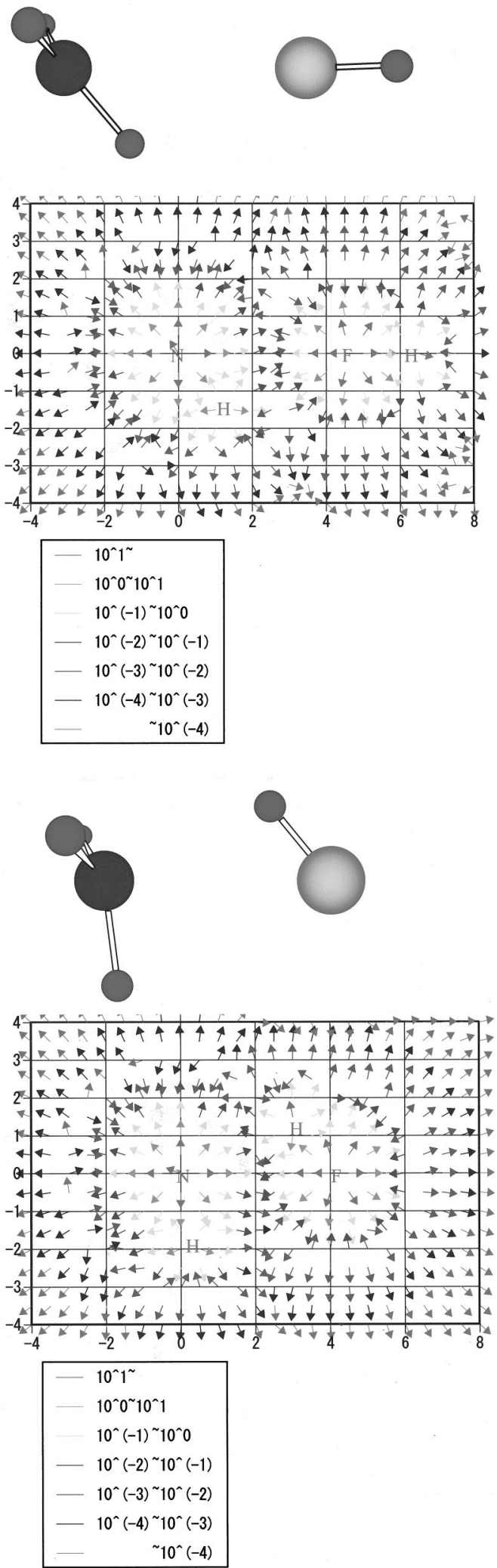

FIG. 4. Characteristic densities are demonstrated for a hydrogen abstraction reaction of $\mathrm{NH}_{3}^{+}$from $\mathrm{NH}_{3}$ along the IRC. For other details, see the caption of Fig. 2. 


$$
\mathrm{NH}_{3}{ }^{+}+\mathrm{HF} \rightarrow \mathrm{NH}_{4}^{+}+\mathrm{F}
$$

\section{Transition State}

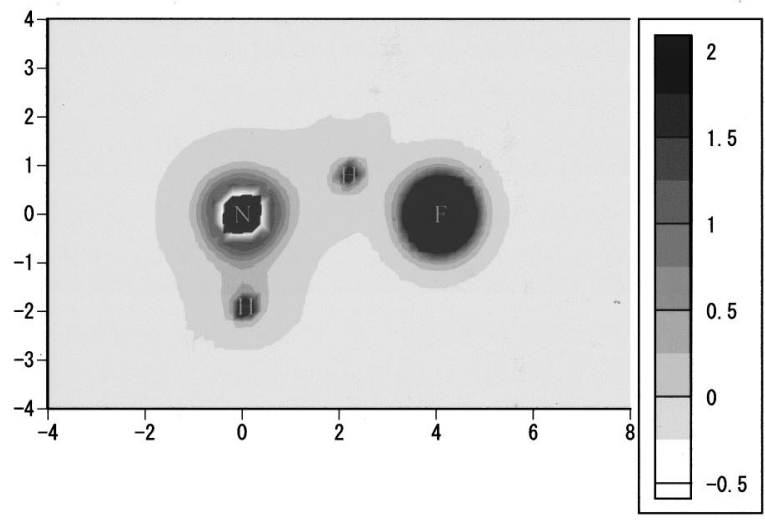

(c)

$$
\mathrm{NH}_{3}{ }^{+}+\mathrm{HF} \rightarrow \mathrm{NH}_{4}^{+}+\mathrm{F}
$$

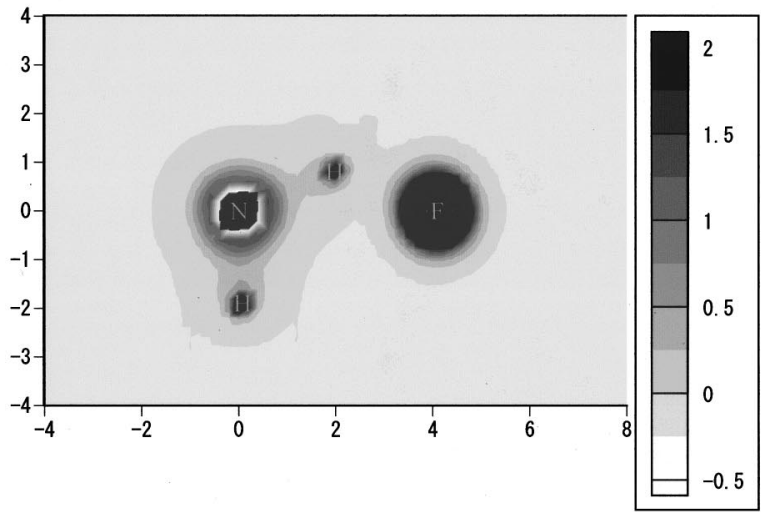

(b)
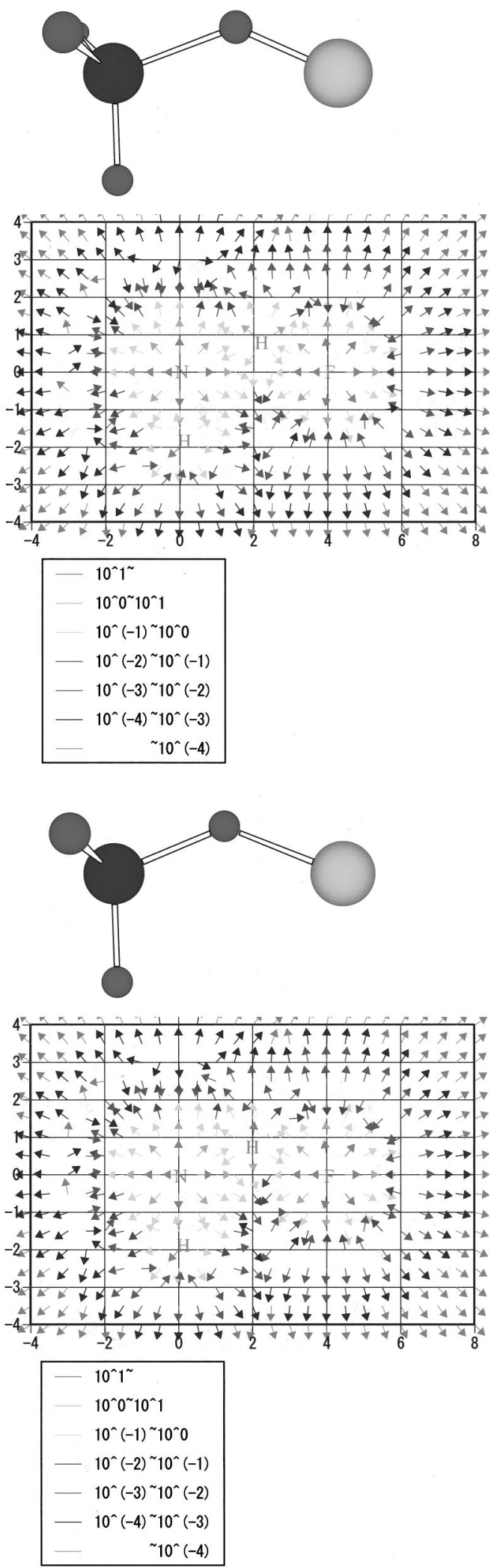

FIG. 4. (Continued.)

ing reaction system is anticipated and actually found, in which, however, at the reactant cell, (b), the $\mathrm{N}-\mathrm{N}$ bond is slightly broken because the migrating $\mathrm{H}$ atom should rotate around the $\mathrm{N}$ pivot center, and further at the transition state, (b), the kinetic energy density $n_{T}(\vec{r})$ merges again at the corner of $\mathrm{H}$ to form the new $\mathrm{H}-\mathrm{N}$ bond. This recoalescence dictates the electronic transition state now at the saddle point on the potential energy surface. 


$$
\mathrm{NH}_{3}{ }^{+}+\mathrm{HF} \rightarrow \mathrm{NH}_{4}^{+}+\mathrm{F}
$$

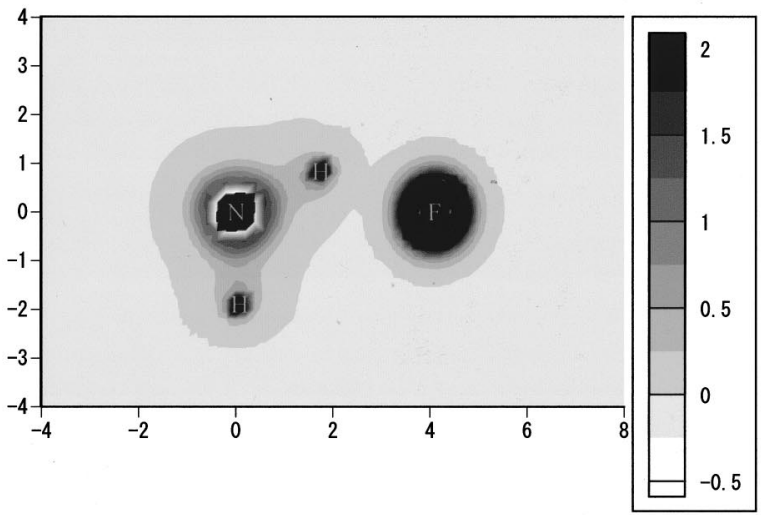

(e)
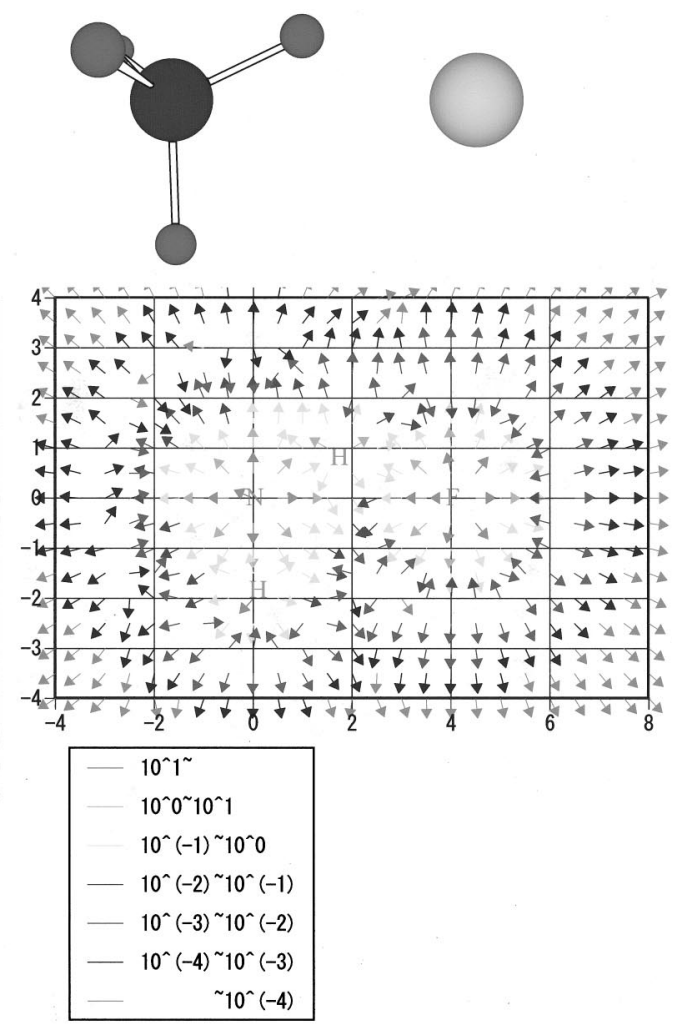

FIG. 4. (Continued.)

\section{CONCLUSION}

The new regional energy decomposition scheme in Refs. 7 and 8 has been extended to an infinitely small regional energy decomposition scheme, namely, the electronic energy density decomposition scheme. The energy of chemical reaction is visualized in real space using the electronic energy density $n_{E}(\vec{r})$ associated with the electron density $n(\vec{r})$. The $n_{E}(\vec{r})$ is decomposed into the kinetic energy density $n_{T}(\vec{r})$, the one-electronic potential energy density $n_{V}(\vec{r})$, the twoelectronic potential energy density $n_{W}(\vec{r})$. The kinetic energy density $n_{T}(\vec{r})$ is used to identify the intrinsic shape of the reactants, the electronic transition state and the reaction products along the course of the chemical reaction coordinate. The intrinsic shape is identified with the electronic interface $S$ that discriminates the region of the electronic drop $R_{D}$ from the region of the electronic atmosphere $R_{A}$ in the density distribution of the electron gas. Using the $n_{E}(\vec{r})$, we can pick up any point in a chemical reaction system and find how the electronic energy $E$ is assigned to the selected point. We have realized the Coulson conjecture "the physical meaning of the probability density should be related with the energy density" 4 in such a way that the quantum electronic energy density $n_{E}(\vec{r})$ is manifestly given as the exact functional of electron density $n(\vec{r})$. We can then integrate the $n_{E}(\vec{r})$ in any region $R$ surrounding the point and find out the regional electronic energy contribution $E_{R}$ to the global $E$. The $E_{R}$ in thermodynamic ensemble without radiation field is realized in electrochemistry as the intrinsic Volta electric potential $\phi_{R}$ and the intrinsic Herring-Nichols work function $\Phi_{R}$.
We have picked up first a hydrogen-like atom for which we have analytical exact expressions of the relativistic kinetic energy density and its nonrelativistic version $n_{T}(\vec{r})$. These expressions are valid for any excited bound states as well as the ground state. For the $\mathrm{H}$ atom we have shown the analytical exact expression and found that the intrinsic shape of $\mathrm{H}$ atom of radius 2 a.u. $\approx 1.058 \AA$ appears to be comparable to the standard atomic radius 1.5 a.u. $\approx 0.79 \AA$ of $\mathrm{H}$ atom reduced by a factor of $75 \%$. For He atom the $R_{D}$ is the sphere surrounded by the $S$ of radius $\approx 0.676 \AA$. This intrinsic shape of He atom is comparable to the standard atomic radius $0.49 \AA$ of $\mathrm{He}$ atom reduced by a factor of $\approx 72 \%$. The smaller shape as compared with the $\mathrm{H}$ atom is well reproduced together with similar value of the reduction factor. For a simple chemical reaction system $\mathrm{He}+\mathrm{H}^{+}$, the polarization and the accumulation of electron density in the course of the reaction coordinate has been clearly demonstrated in terms of the $R_{D}$ and $S$, which leads to the shape of the intrinsic electronic transition state and finally to the molecular shape of the $\mathrm{HeH}^{+}$complex as the reaction product.

We have selected following five reaction systems and shown the figures of the $n_{T}(\vec{r})$ as well as the other energy densities along the intrinsic reaction coordinates (IRCs): a protonation reaction to $\mathrm{He}$, addition reactions of $\mathrm{HF}$ to $\mathrm{C}_{2} \mathrm{H}_{4}$ and $\mathrm{C}_{2} \mathrm{H}_{2}$, hydrogen abstraction reactions of $\mathrm{NH}_{3}^{+}$from $\mathrm{HF}$ and $\mathrm{NH}_{3}$. Valence electrons possess their unique delocalized drop region remote from those heavily localized drop regions adhered to core electrons. The kinetic energy density $n_{T}(\vec{r})$ and the tension density $\vec{\tau}^{S}(\vec{r})$ can vividly demonstrate the formation of chemical bond. Various basic chemical concepts 
in these chemical reaction systems have been clearly visualized in real three-dimensional space.

We have demonstrated the new concept of the electronic energy density using simple atom and chemical reaction sys- tems. The application of the present theory to various chemical reaction systems is under way: excited Li cluster formation reactions, ${ }^{20} \mathrm{~N} 2{ }^{+}-\mathrm{O} 2$ charge transfer reactions, ${ }^{21} \mathrm{GaN}$ crystal growth gas phase and surface reactions, ${ }^{22}$ reactions of

$$
\mathrm{NH}_{3}{ }^{+}+\mathrm{NH}_{3} \rightarrow \mathrm{NH}_{4}{ }^{+}+\mathrm{NH}_{2}
$$

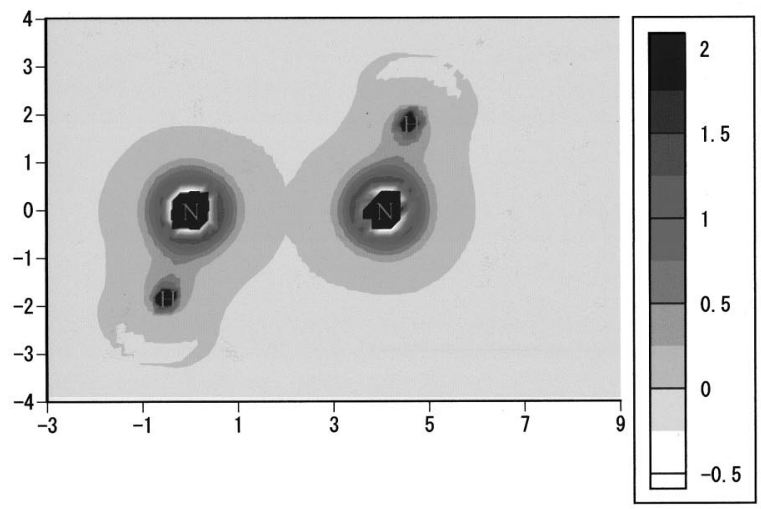

(a)

$$
\mathrm{NH}_{3}{ }^{+}+\mathrm{NH}_{3} \rightarrow \mathrm{NH}_{4}^{+}+\mathrm{NH}_{2}
$$

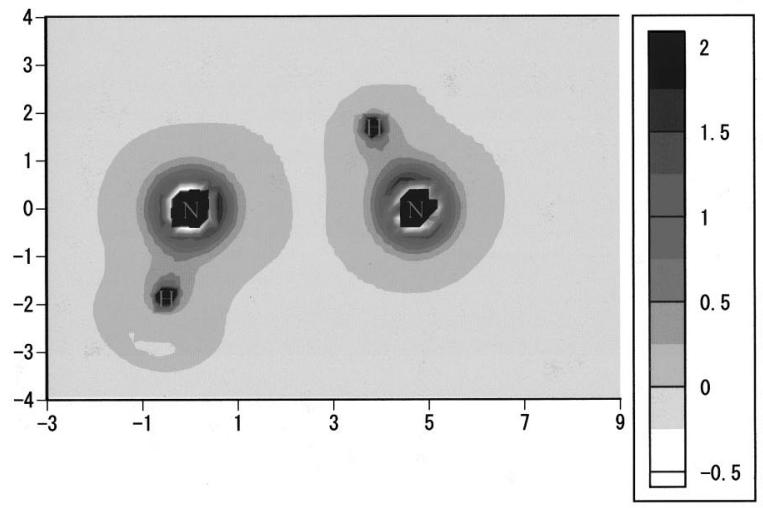

(b)
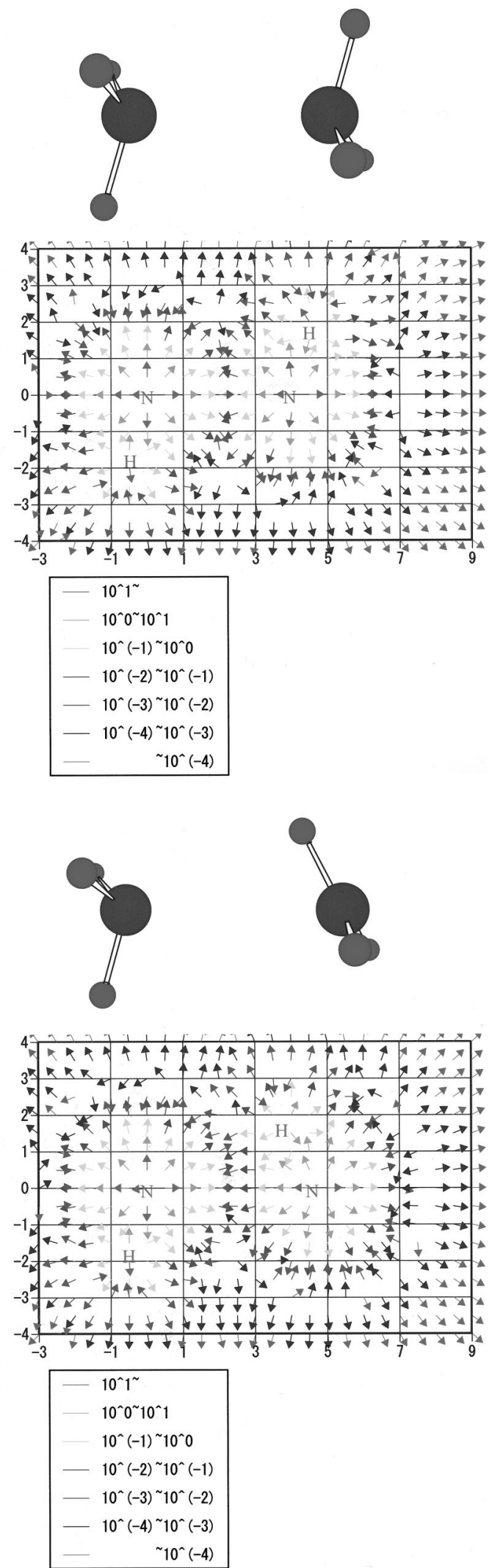

FIG. 5. Characteristic densities are demonstrated for a hydrogen abstraction reaction of $\mathrm{NH}_{3}^{+}$from $\mathrm{HF}$ along the IRC. For other details, see the caption of Fig. 2. 


$$
\mathrm{NH}_{3}{ }^{+}+\mathrm{NH}_{3} \rightarrow \mathrm{NH}_{4}^{+}+\mathrm{NH}_{2}
$$

\section{Transition State}

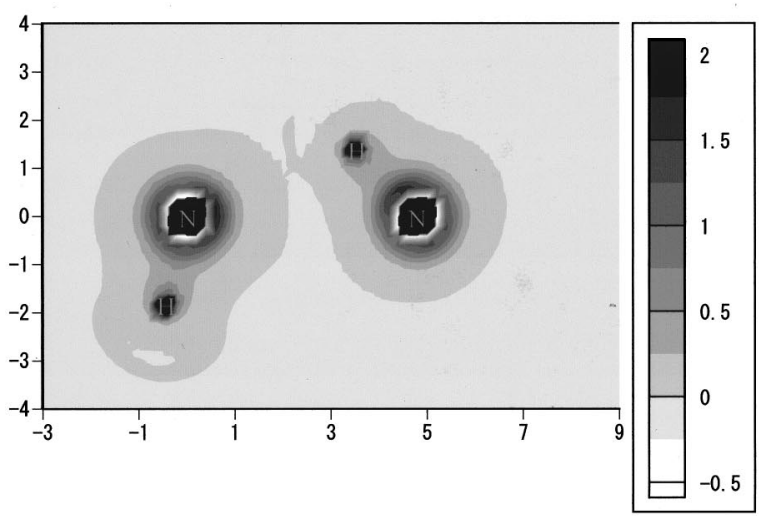

(c)

$$
\mathrm{NH}_{3}{ }^{+}+\mathrm{NH}_{3} \rightarrow \mathrm{NH}_{4}{ }^{+}+\mathrm{NH}_{2}
$$

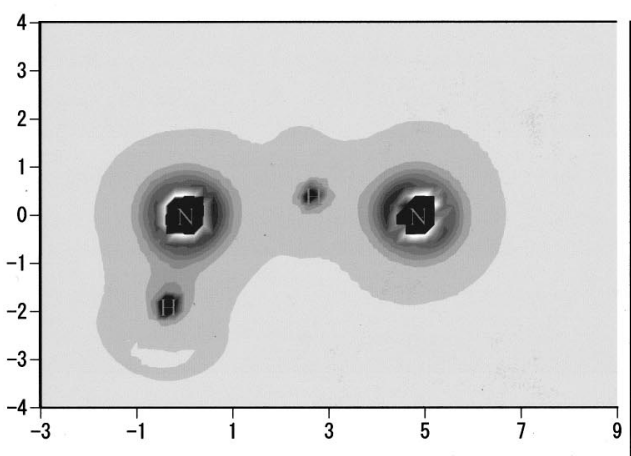

(d)

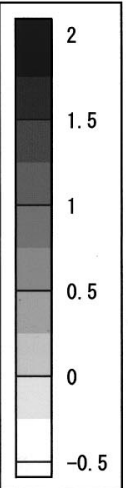

FIG. 5. (Continued.)
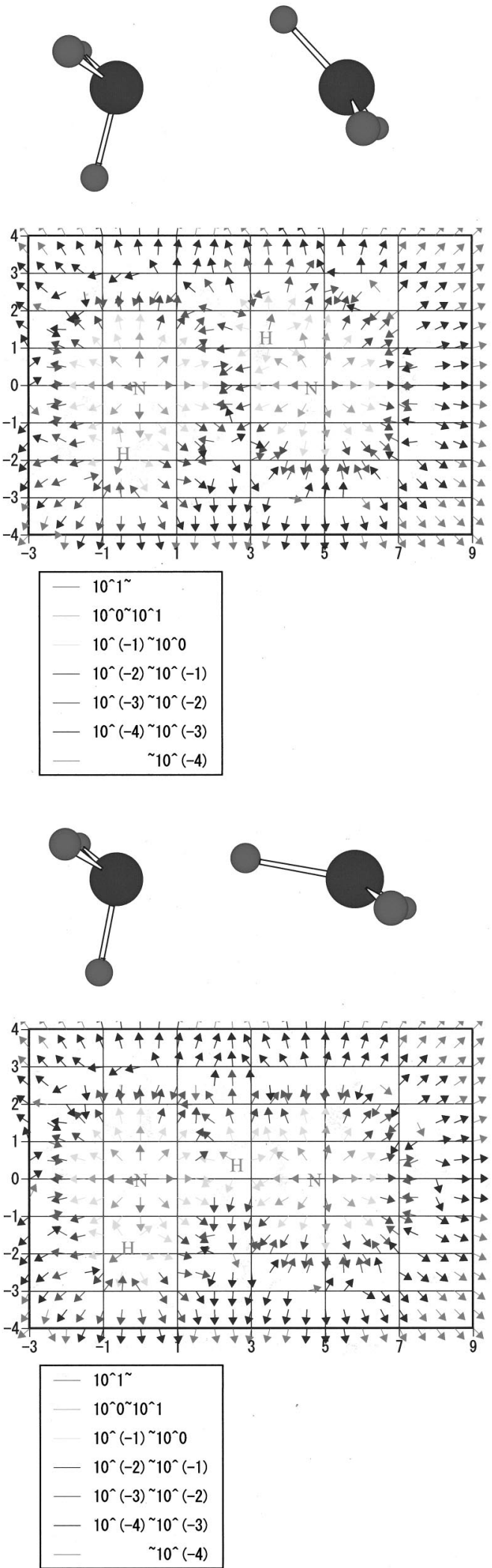

$\mathrm{F}$ radicals and ions with hydrogen-terminated $\mathrm{Si}(111)$ thin film, ${ }^{23}$ etching reactions of $\operatorname{TiN}(111)$ surface with $\mathrm{F}$ radicals, ${ }^{24}$ electromigration reactions of $\mathrm{Al}$ crystal, ${ }^{25}$ reactions of $\mathrm{O}$ radicals and ions with hydrogen-terminated
Si(111) thin film, ${ }^{26}$ chemical potential inequality principle and inhomogeneity of work function. ${ }^{27}$

Detailed relativistic treatments of the gauges, antiparticles and renormalizations will be published elsewhere. ${ }^{28}$ 


$$
\mathrm{NH}_{3}^{+}+\mathrm{NH}_{3} \rightarrow \mathrm{NH}_{4}^{+}+\mathrm{NH}_{2}
$$
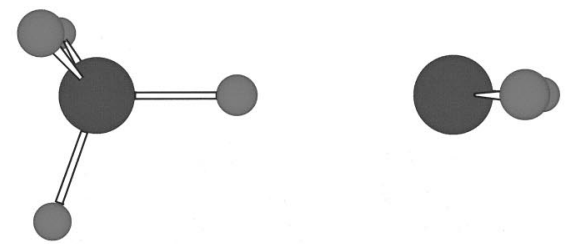

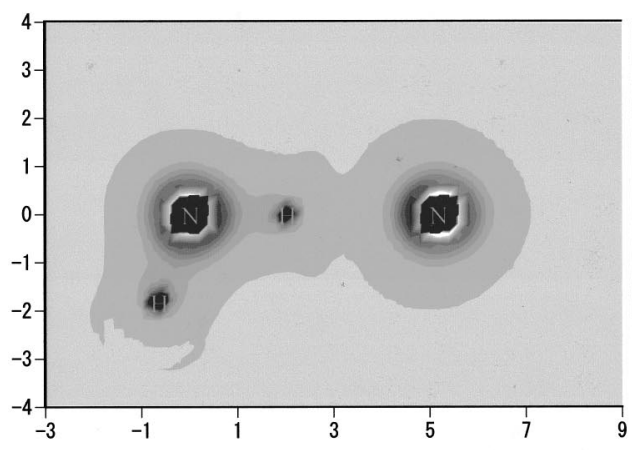

(e)

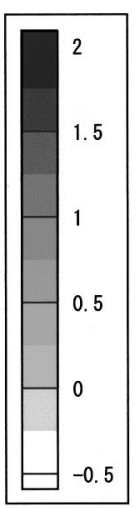

FIG. 5. (Continued.)

5. (Continued)

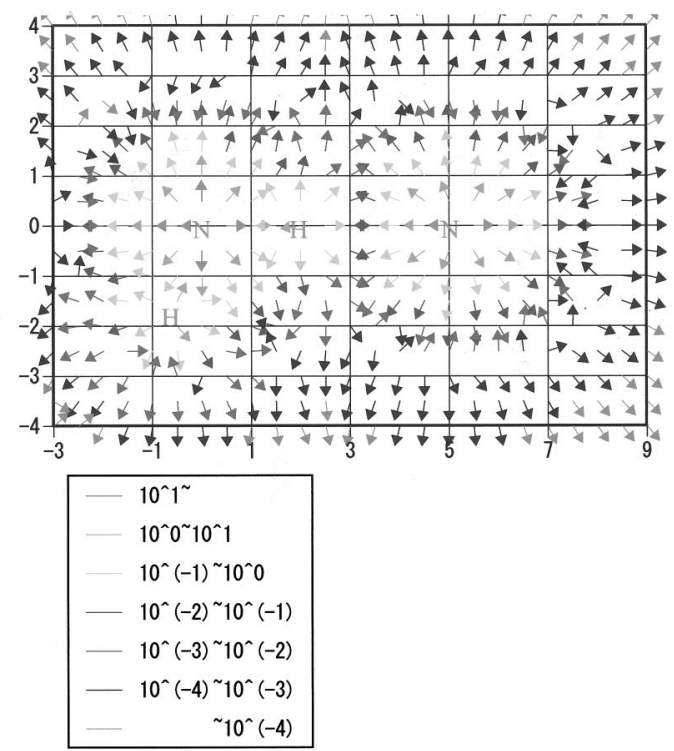

$10(-4)$

\section{ACKNOWLEDGMENT}

This work was supported by a Grant-in-Aid for Scientific Research from the Ministry of Education, Science and Culture of Japan.

\section{APPENDIX A}

In quantum electrodynamics (QED), the space-time coordinates $x^{\mu}$ means

$$
x^{\mu}=\left(x^{0}, x^{k}\right)=\left(x^{0}, x^{1}, x^{2}, x^{3}\right)=(c t, x, y, z)=(c t, \vec{r}),
$$

where Greek letter runs from 0 to $3, \mu=0,1,2,3$, and the italic letter from 1 to $3, k=1,2,3$. Einstein summation convention for duplicate indices is used throughout in this paper. The space-time coordinates $x^{\mu}$ may be suppressed to appear unless otherwise stated explicitly so that $f$ or $f(\vec{r})$ may stand for $f(t, \vec{r})$. The contravariant vector $a^{\mu}$ is transformed to the covariant vector $a_{\mu}$ through

$$
a_{\mu}=g_{\mu \nu} a^{\nu},
$$

where the metric tensor is given as

$$
g_{\mu \nu}=\left(\begin{array}{cccc}
1 & 0 & 0 & 0 \\
0 & -1 & 0 & 0 \\
0 & 0 & -1 & 0 \\
0 & 0 & 0 & -1
\end{array}\right)=g^{\mu \nu}
$$

The gradient vectors stand for

$$
\begin{aligned}
\partial_{\mu}=\frac{\partial}{\partial x^{\mu}} & =\left(\frac{\partial}{\partial x^{0}}, \frac{\partial}{\partial x^{1}}, \frac{\partial}{\partial x^{2}}, \frac{\partial}{\partial x^{3}}\right) \\
& =\left(\frac{1}{c} \frac{\partial}{\partial t}, \vec{\nabla}\right)=\left(\frac{1}{c} \frac{\partial}{\partial t}, \operatorname{grad}\right), \\
\partial^{\mu}=g^{\mu \nu} \partial_{\nu} & =\left(\frac{\partial}{\partial x^{0}},-\frac{\partial}{\partial x^{1}},-\frac{\partial}{\partial x^{2}},-\frac{\partial}{\partial x^{3}}\right) \\
& =\left(\frac{1}{c} \frac{\partial}{\partial t},-\vec{\nabla}\right)=\left(\frac{1}{c} \frac{\partial}{\partial t},-\operatorname{grad}\right) .
\end{aligned}
$$

The gauge potential operators stand for

$$
\begin{aligned}
& \hat{A}^{\mu}(\vec{r})=(\hat{\phi}(\vec{r}), \hat{\vec{A}}(\vec{r})), \\
& \hat{A}_{\mu}(\vec{r})=(\hat{\phi}(\vec{r}),-\hat{\vec{A}}(\vec{r})) .
\end{aligned}
$$

The covariant derivative operators with gauge potential operator $\hat{A}_{\mu}(\vec{r})$ :

$$
\begin{aligned}
& \hat{D}_{\mu}(\vec{r})=\partial_{\mu}-i \frac{e}{\hbar c} \hat{A}_{\mu}(\vec{r}), \\
& \hat{D}_{\mu}^{+}(\vec{r})=\partial_{\mu}+i \frac{e}{\hbar c} \hat{A}_{\mu}(\vec{r}),
\end{aligned}
$$

satisfy

$$
\hat{D}_{\mu}(\vec{r}) \hat{D}_{\nu}(\vec{r})-\hat{D}_{\nu}(\vec{r}) \hat{D}_{\mu}(\vec{r})=-i \frac{e}{\hbar c} \hat{F}_{\mu \nu}(\vec{r}),
$$




$$
\hat{D}_{\mu}^{+}(\vec{r}) \hat{D}_{\nu}^{+}(\vec{r})-\hat{D}_{\nu}^{+}(\vec{r}) \hat{D}_{\mu}^{+}(\vec{r})=i \frac{e}{\hbar c} \hat{F}_{\mu \nu}(\vec{r}),
$$

with the gauge field operators $\hat{F}_{\mu \nu}(\vec{r})$ defined as

$$
\begin{aligned}
\hat{F}_{\mu \nu}(\vec{r}) & =\partial_{\mu} \hat{A}_{\nu}(\vec{r})-\partial_{\nu} \hat{A}_{\mu}(\vec{r}) \\
& =\left(\begin{array}{cccc}
0 & \hat{E}_{x}(\vec{r}) & \hat{E}_{y}(\vec{r}) & \hat{E}_{z}(\vec{r}) \\
-\hat{E}_{x}(\vec{r}) & 0 & -\hat{B}_{z}(\vec{r}) & \hat{B}_{y}(\vec{r}) \\
-\hat{E}_{y}(\vec{r}) & \hat{B}_{z}(\vec{r}) & 0 & -\hat{B}_{x}(\vec{r}) \\
-\hat{E}_{z}(\vec{r}) & -\hat{B}_{y}(\vec{r}) & \hat{B}_{x}(\vec{r}) & 0
\end{array}\right) .
\end{aligned}
$$

Then, the electric field operator $\widehat{\vec{E}}(\vec{r})$ and the magnetic field operator $\hat{\vec{B}}(\vec{r})$ are represented using the gauge potential operators as

$$
\begin{aligned}
& \hat{\vec{E}}(\vec{r})=-\operatorname{grad} \hat{\phi}(\vec{r})-\frac{1}{c} \frac{\partial \hat{\vec{A}}(\vec{r})}{\partial t}, \\
& \hat{\vec{B}}(\vec{r})=\operatorname{rot} \hat{\vec{A}}(\vec{r}), \quad \operatorname{div} \hat{\vec{A}}(\vec{r})=0,
\end{aligned}
$$

and satisfy the Maxwell's equations as follows:

$$
\begin{aligned}
& \operatorname{rot} \hat{\vec{E}}(\vec{r})+\frac{1}{c} \frac{\partial \hat{\vec{B}}(\vec{r})}{\partial t}=0, \\
& \operatorname{div} \hat{\vec{B}}(\vec{r})=0, \\
& \operatorname{div} \hat{\vec{E}}(\vec{r})=4 \pi \hat{\rho}(\vec{r}), \\
& \operatorname{rot} \hat{\vec{B}}(\vec{r})-\frac{1}{c} \frac{\partial \hat{\vec{E}}(\vec{r})}{\partial t}=\frac{4 \pi \hat{\vec{j}}(\vec{r}),}{c}
\end{aligned}
$$

where $\hat{\rho}(\vec{r})$ is the electronic charge density operator and $\hat{\vec{j}}(\vec{r})$ is the electronic current density operator [see the text: specifically Eqs. (36) and (37)]. The Hamiltonian density operator of the electromagnetic field $\hat{H}_{\mathrm{EM}}(\vec{r})$ is represented as follows:

$$
\hat{H}_{\mathrm{EM}}(\vec{r})=\frac{1}{8 \pi}\left(\hat{\vec{E}}^{2}(\vec{r})+\widehat{\vec{B}}^{2}(\vec{r})\right)-\hat{\phi}(\vec{r}) \hat{\rho}(\vec{r}) .
$$

The Hamiltonian operator of the field is

$$
\hat{H}_{\mathrm{EM}}=\int d^{3} \vec{r} \hat{H}_{\mathrm{EM}}(\vec{r}) \text {. }
$$

The radiation field is a particular electromagnetic field with neither macroscopic charge density nor charge current density. In the classical treatment of the radiation field, the energy density $H_{\text {radiation }}(\vec{r})$ is obtained as

$$
H_{\text {radiation }}(\vec{r})=\frac{1}{8 \pi}\left(\vec{E}^{2}(\vec{r})+\vec{B}^{2}(\vec{r})\right),
$$

which leads to the total energy $H_{\text {radiation }}$ of the radiation field as

$$
H_{\text {radiation }}=\int d^{3} \vec{r} H_{\text {radiation }}(\vec{r}) \text {. }
$$

Maxwell's equations are solved using gauge potential $\vec{A}(\vec{r})$ as

$$
\vec{E}(\vec{r})=-\frac{1}{c} \frac{\partial \vec{A}(\vec{r})}{\partial t}, \quad \vec{B}(\vec{r})=\operatorname{rot} \vec{A}(\vec{r}), \quad \operatorname{div} \vec{A}(\vec{r})=0,
$$

with

$$
\partial_{\mu} \partial^{\mu} \vec{A}(\vec{r})=0, \quad \partial_{\mu} \partial^{\mu}=\frac{1}{c^{2}}\left(\frac{\partial}{\partial t}\right)^{2}-\Delta,
$$

where d'Alembertian $\partial_{\mu} \partial^{\mu}$ is written using Laplacian $\Delta$ :

$$
\Delta=\partial_{k}^{2} .
$$

\section{APPENDIX B}

In quantum electrodynamics (QED), the Hamiltonian density operator $\hat{H}_{\mathrm{QED}}(\vec{r})$ is represented as follows:

$$
\hat{H}_{\mathrm{QED}}(\vec{r})=\hat{H}_{\mathrm{EM}}(\vec{r})+\hat{H}_{\mathrm{Dirac}}(\vec{r}),
$$

where $\hat{H}_{\mathrm{EM}}(\vec{r})$ is the electromagnetic Hamiltonian density operator [see Appendix A: specifically Eq. (A19)] and $\hat{H}_{\text {Dirac }}(\vec{r})$ is the Dirac electronic Hamiltonian density operator:

$$
\hat{H}_{\text {Dirac }}(\vec{r})=\hat{\psi}^{+}(\vec{r}) \hat{h}_{\text {Dirac }}(\vec{r}) \hat{\psi}(\vec{r}),
$$

with

$$
\hat{h}_{\text {Dirac }}(\vec{r})=-i \hbar c \gamma^{0} \gamma^{k} \hat{D}_{k}(\vec{r})+\gamma^{0} m c^{2}-e \hat{\phi}(\vec{r}),
$$

and $\gamma^{\mu}=\left(\gamma^{0}, \vec{\gamma}\right)$ are the Dirac matrices.

The electronic four-dimensional four-component spinor operator,

$$
\hat{\psi}(\vec{r})=\left(\begin{array}{c}
\hat{\psi}_{1}(\vec{r}) \\
\hat{\psi}_{2}(\vec{r}) \\
\hat{\psi}_{3}(\vec{r}) \\
\hat{\psi}_{4}(\vec{r})
\end{array}\right),
$$

satisfies the canonical anticommutation relationships,

$$
\begin{aligned}
& \hat{\psi}_{\mu}(\vec{r}) \hat{\psi}_{\nu}^{+}(\vec{s})+\hat{\psi}_{\nu}^{+}(\vec{s}) \hat{\psi}_{\mu}(\vec{r})=\delta_{\mu}^{\nu} \delta(\vec{r}-\vec{s}), \\
& \hat{\psi}_{\mu}(\vec{r}) \hat{\psi}_{\nu}(\vec{s})+\hat{\psi}_{\nu}(\vec{s}) \hat{\psi}_{\mu}(\vec{r})=0, \\
& \hat{\psi}_{\mu}^{+}(\vec{r}) \hat{\psi}_{\nu}^{+}(\vec{s})+\hat{\psi}_{\mu}^{+}(\vec{r}) \hat{\psi}_{\nu}^{+}(\vec{s})=0 .
\end{aligned}
$$

Using the Hamiltonian operator,

$$
\hat{H}_{\mathrm{QED}}=\int d^{3} \vec{r} \hat{H}_{\mathrm{QED}}(\vec{r}),
$$

the equation of motion is found to be

$$
i \hbar \frac{\partial}{\partial t} \hat{\psi}(\vec{r})=\left[\hat{\psi}(\vec{r}), \hat{H}_{\mathrm{QED}}\right]=\hat{h}_{\mathrm{Dirac}}(\vec{r}) \hat{\psi}(\vec{r}) .
$$

Equivalently, we get

$$
i \hbar \gamma^{\mu} \hat{D}_{\mu}(\vec{r}) \hat{\psi}(\vec{r})-m c \hat{\psi}(\vec{r})=0,
$$

and similarly 


$$
-i \hbar \hat{D}_{\mu}^{+}(\vec{r}) \hat{\bar{\psi}}(\vec{r}) \gamma^{\mu}-m c \hat{\bar{\psi}}(\vec{r})=0,
$$

where $\hat{\bar{\psi}}(\vec{r})$ is the Dirac conjugate to $\hat{\psi}(\vec{r})$ :

$$
\hat{\bar{\psi}}(\vec{r})=\hat{\psi}^{+}(\vec{r}) \gamma^{0} .
$$

The theory is invariant under gauge transformation as follows:

$$
\begin{aligned}
& \hat{\psi}(\vec{r}) \rightarrow \hat{\psi}^{\prime}(\vec{r})=\exp \left(-i \frac{e}{\hbar c} \hat{\theta}(\vec{r})\right) \hat{\psi}(\vec{r}), \\
& \hat{A}_{\mu}(\vec{r}) \rightarrow \hat{A}_{\mu}^{\prime}(\vec{r})=\hat{A}_{\mu}(\vec{r})-\partial_{\mu} \hat{\theta}(\vec{r}), \quad \Delta \hat{\theta}(\vec{r})=0 .
\end{aligned}
$$

In the nonrelativistic treatment, the electronic field operator is $\hat{\chi}(\vec{r})$ which denotes the three-dimensional twocomponent spinor,

$$
\hat{\chi}(\vec{r})=\left(\begin{array}{c}
\hat{\chi}^{1}(\vec{r}) \\
\hat{\chi}^{2}(\vec{r})
\end{array}\right)
$$

which satisfies the canonical anticommutation relationships,

$$
\begin{aligned}
& \hat{\chi}(\omega) \hat{\chi}^{+}\left(\omega^{\prime}\right)+\hat{\chi}^{+}\left(\omega^{\prime}\right) \hat{\chi}(\omega)=\delta\left(\omega-\omega^{\prime}\right), \\
& \hat{\chi}(\omega) \hat{\chi}\left(\omega^{\prime}\right)+\hat{\chi}\left(\omega^{\prime}\right) \hat{\chi}(\omega)=0, \\
& \hat{\chi}^{+}(\omega) \hat{\chi}^{+}\left(\omega^{\prime}\right)+\hat{\chi}^{+}\left(\omega^{\prime}\right) \hat{\chi}^{+}(\omega)=0,
\end{aligned}
$$

where, for the sake of simplicity of presentation, Cartesian and spin variables altogether are represented by $\omega$ as

$$
\omega=(\vec{r}, s), \quad s=1,2 .
$$

Using the nonrelativistic Hamiltonian operator

$$
\hat{H}=\int d^{3} \vec{r} \hat{H}(\vec{r}),
$$

with the classical treatment of the radiation field, the equation of motion is found to be

$$
\begin{aligned}
i \hbar \frac{\partial}{\partial t} \hat{\chi}(\vec{r})=[\hat{\chi}(\vec{r}), \hat{H}]= & -\frac{\hbar^{2}}{2 m} D_{k}^{2}(\vec{r}) \hat{\chi}(\vec{r})+\nu(\vec{r}) \hat{\chi}(\vec{r}) \\
& +\int d^{3} \vec{s} \hat{\chi}^{+}(\vec{s}) w(\vec{r}, \vec{s}) \hat{\chi}(\vec{s}) \hat{\chi}(\vec{r}),
\end{aligned}
$$

and similarly,

$$
\begin{aligned}
-i \hbar \frac{\partial}{\partial t} \hat{\chi}^{+}(\vec{r})= & -\frac{\hbar^{2}}{2 m} D_{k}^{* 2}(\vec{r}) \hat{\chi}^{+}(\vec{r})+\nu(\vec{r}) \hat{\chi}^{+}(\vec{r}) \\
& +\hat{\chi}^{+}(\vec{r}) \int d^{3} \vec{s} \hat{\chi}^{+}(\vec{s}) w(\vec{r}, \vec{s}) \hat{\chi}(\vec{s}) .
\end{aligned}
$$

The state vector in the nonrelativistic Fock space is represented as

$$
|\Psi\rangle=\sum_{N=0}^{\infty} \int d^{4} \omega_{1} \cdots d^{4} \omega_{N}\left|\omega_{1}, \ldots, \omega_{N}\right\rangle \Phi_{N}\left(\omega_{1}, \ldots, \omega_{N}\right),
$$

$\left|\omega_{1}, \ldots, \omega_{N}\right\rangle=\frac{1}{\sqrt{N !}} \hat{\chi}^{+}\left(\omega_{1}\right) \cdots \hat{\chi}^{+}\left(\omega_{N}\right)|0\rangle$,

$$
\begin{aligned}
& \left\langle\omega_{1}, \ldots, \omega_{N} \mid \omega_{1}^{\prime}, \ldots, \omega_{M}^{\prime}\right\rangle \\
& \quad=\delta_{N M} \frac{1}{N !} \sum_{\sigma}(-)^{\sigma} \delta\left(\omega_{1}-\omega_{\sigma 1}^{\prime}\right) \cdots \delta\left(\omega_{N}-\omega_{\sigma N}^{\prime}\right),
\end{aligned}
$$

where $\Phi_{N}\left(\omega_{1}, \ldots, \omega_{N}\right)$ denotes the antisymmetric wave function in the N-electron nonrelativistic Hilbert space and $\Sigma_{\sigma}(-)^{\sigma}$ denotes antisymmetric summation over every possible permutation $\sigma$ of numbers $1, \ldots, N$. The nonrelativistic Schrödinger equation is written as

$$
i \hbar \frac{\partial}{\partial t}|\Psi\rangle=\hat{H}|\Psi\rangle \text {. }
$$

${ }^{1}$ K. Fukui, J. Phys. Chem. 74, 4161 (1970); Acc. Chem. Res. 14, 363 (1981).

${ }^{2}$ A. Tachibana, J. Math. Chem. 7, 95 (1991); in Conceptual Trends in Quantum Chemistry, edited by E. S. Kryachko and J. L. Calais (Kluwer, Dordrecht, 1994), pp. 101-118.

${ }^{3}$ A. Tachibana, in The Transition State - A Theoretical Approach, edited by T. Fueno (Kodansha, Tokyo, 1999), pp. 217-247.

${ }^{4}$ C. A. Coulson, Valence, 2nd. ed. (Clarendon, Oxford, 1961).

${ }^{5}$ I. Fleming, Frontier Orbitals and Organic Chemical Reactions (Wiley, New York, 1976).

${ }^{6}$ A. Szabo and N. S. Ostlund, Modern Quantum Chemistry (Macmillan, New York, 1982)

${ }^{7}$ A. Tachibana, Theor. Chem. Acc. 102, 188 (1999).

${ }^{8}$ A. Tachibana, Int. J. Quantum Chem., Quantum Chem. Symp. 21, 181 (1987).

${ }^{9}$ A. Tachibana, Int. J. Quantum Chem. 57, 423 (1996); A. Tachibana and R. G. Parr, ibid. 41, 527 (1992).

${ }^{10}$ A. Tachibana, K. Nakamura, K. Sakata, and T. Morisaki, Int. J. Quantum Chem. 74, 669 (1999).

${ }^{11}$ P. Hohenberg and W. Kohn, Phys. Rev. 136, B864 (1964).

${ }^{12}$ N. D. Mermin, Phys. Rev. A 137, 1441 (1965).

${ }^{13}$ R. G. Parr and W. Yang, Density Functional Theory of Atoms and Molecules (Oxford University Press, Oxford, 1989).

${ }^{14}$ T. Kato, Commun. Pure Appl. Math. 10, 151 (1957).

${ }^{15}$ W. A. Bingel, Z. Naturforsch. A 18A, 1249 (1963); Theor. Chim. Acta 8, 54 (1967)

${ }^{16}$ R. T Pack and W. B. Brown, J. Chem. Phys. 45, 556 (1966).

${ }^{17}$ C. Herring and M. H. Nichols, Rev. Mod. Phys. 21, 185 (1949).

${ }^{18}$ E. Wigner and J. Bardeen, Phys. Rev. 48, 84 (1935).

${ }^{19}$ A. Tachibana, T. Suzuki, N. Yoshida, Y. Teramoto, and T. Yamabe, Chem. Phys. 156, 79 (1991); A. Tachibana, S. Kawauchi, Y. Kurosaki, N. Yoshida, T. Ogihara, and T. Yamabe, J. Phys. Chem. 95, 9647 (1991); A. Tachibana, T. Suzuki, Y. Teramoto, N. Yoshida, T. Sato, and T. Yamabe, J. Chem. Phys. 95, 4136 (1991); A. Tachibana, T. Suzuki, and T. Yamabe, ibid. 97, 4921 (1992); A. Tachibana, S. Kawauchi, K. Nakamura, and H. Inaba, Int. J. Quantum Chem. 57, 673 (1996); A. Tachibana and M. Fuju, J. Chem. Phys. 110, 2323 (1999).

${ }^{20}$ S. Hotta, K. Nakamura, and A. Tachibana, in the Abstract of the 2000 Symposium on Molecular Science, Bunshikouzou Sougou Touronkai, (in Japanese), Tokyo, Japan, 27-30 September 2000, No. 1p-211.

${ }^{21}$ K. Hasegawa, K. Nakamura, and A. Tachibana, Ref. 20, No. 4p-23; see also A. Tachibana, K. Nakamura, T. Yano, Y. Suguyama, and S. Tanimura, J. Phys. Chem. 103, 5749 (1999).

${ }^{22}$ M. Ikenaga, K. Nakamura, and A. Tachibana, in the Abstract of the 2000 Symposium on Molecular Science, Bunshikouzou Sougou Touronkai (in Japanese), Tokyo, Japan, 27-30 September 2000, No. 1p-181; T. Hayashi, K. Nakamura, and A. Tachibana, in the Abstract of the 61st Annual Symposium of the Japanese Society of the Applied Physics, Sapporo, Japan, 3-7 September 2000, No. 3a-Y-1; see also A. Tachibana, K. Nakamura, O. Makino, H. Tokunaga, N. Akutsu, and K. Matsumoto, in Proceedings of the Second International Symposium on Blue Laser and Light Emitting Diodes (Ohmsha, Tokyo, 1998), pp. 308-309; A. Tachibana, O. Makino, S. Tanimura, H. Tokunaga, N. Akutsu, and K. Matsumoto, Phys. Status Solidi B 176, 699 (1999); O. Makino, K. Nakamura, A. Tachibana, H. Tokunaga, N. Akutsu, and K. Matsumoto, Appl. Surf. Sci. 159/160, 374 (2000); K. Nakamura, O. Makino, A. Tachibana, and K. Matsumoto, J. Organomet. Chem. 611, 514 (2000). 
${ }^{23}$ M. Ikenaga and A. Tachibana, in the Abstract of the 2000 Symposium on Molecular Science, Bunshikouzou Sougou Touronkai (in Japanese), Tokyo, Japan, 27-30 September 2000, No. 1p-275; O. Makino, K. Sakata, H. Yamazaki, K. Iguchi, and A. Tachibana, in the Abstract of the 61st Annual Symposium of the Japanese Society of the Applied Physics, Sapporo, Japan, 3-7 September 2000, No. 5p-F-3; see also O. Makino, K. Sakata, H. Yamazaki, K. Iguchi, and A. Tachibana, Thin Solid Films 374, 143 (2000); Y. Morikawa, K. Kubota, H. Ogawa, T. Ichiki, A. Tachibana, S. Fujimura, and Y. Horiike, J. Vac. Sci. Technol. A 16, 345 (1998).

${ }^{24}$ Y. Ashida, K. Nakamura, and A. Tachibana, in the Abstract of the 61st Annual Symposium of the Japanese Society of the Applied Physics, Sapporo, Japan, 3-7 September 2000, No. 5p-P8-13; see also A. Tachibana and K. Nakamura, J. Mol. Struct.: THEOCHEM 506, 273 (2000).

${ }^{25} \mathrm{~K}$. Iguchi and A. Tachibana, in the Abstract of the 61st Annual Symposium of the Japanese Society of the Applied Physics, Sapporo, Japan, 3-7 Sep- tember 2000, No. 5a-P8-14; see also K. Iguchi and A. Tachibana, Appl. Surf. Sci. 159/160, 167 (2000).

${ }^{26}$ R. Shinohara, K. Nakamura, and A. Tachibana, in the Abstract of the 61st Annual Symposium of the Japanese Society of the Applied Physics, Sapporo, Japan, 3-7 September 2000), No. 5a-ZC-6; see also A. Tachibana, K. Sakata, and T. Sato, Jpn. J. Appl. Phys., Part 1 37, 4493 (1998); K. Sakata, A. Tachibana, S. Zaima, and Y. Yasuda, ibid. 37, 4962 (1998); K. Sakata, T. Sato, K. Nakamura, A. Osamura, and A. Tachibana, Appl. Surf. Sci. 159/160, 392 (2000).

${ }^{27}$ A. Tachibana, in the Abstract of the 2000 Symposium on Molecular Science, Bunshikouzou Sougou Touronkai (in Japanese), Tokyo, Japan, 27-30 September 2000, No. 2-B-12; in the Abstract of the PACIFICHEM 2000, Honolulu, Hawaii, 14-19 December 2000, No. 90010407; see also, Ref. 7.

${ }^{28} \mathrm{~A}$. Tachibana (to be published). 